\title{
Mixed Social Optima and Nash equilibrium in Linear-Quadratic-Gaussian Mean-field System
}

\author{
Xinwei Feng ${ }^{1}$, Jianhui Huang ${ }^{2}$ and Zhenghong Qiu ${ }^{3, *}$
}

\begin{abstract}
This paper investigates a class of mixed stochastic linear-quadratic-Gaussian (LQG) social optimization and Nash game in the context of a large-scale system. Two types of interactive agents are involved: a major agent and a large number of weakly-coupled minor agents. All minor agents are cooperative to minimize the social cost as the sum of their individual costs, whereas such social cost is conflictive to that of the major agent. Thus, the major agent and all minor agents are further competitive to reach some nonzero-sum Nash equilibrium. Applying the meanfield approximations and person-by-person optimality, we obtain auxiliary control problems for the major agent and minor agents, respectively. The decentralized social strategy is derived by a class of new consistency condition (CC) system, which consists of mean-field forward-backward stochastic differential equations. The well-posedness of CC system is obtained by the discounting method. The related asymptotic social optimality for minor agents and Nash equilibrium for major-minor agents are also verified.
\end{abstract}

Index Terms-Decentralized control, LQG mean-field strategy, Mean-field forward backward stochastic differential equations, Nash equilibrium, Social-optimality.

\section{INTRODUCTION}

The Mean-field methodology for large-population systems has been extensively studied recently. The central goal of an individual agent in the large-population system is to obtain decentralized strategy based on its own limited information. One efficient approach is the mean-field method which enables us to obtain the decentralized strategy through the limiting auxiliary control problem and the related consistency condition (CC) system. Along this direction, the interested readers are referred to [15], [17] and [18] for the derivation of mean-field games, [2], [10], [13] for linear-quadratic-Gaussian (LQG) mean-field games, [3] for probabilistic analysis in meanfield games, [21] for risk-sensitive mean-field games, [20] for discrete-time mean-field games. [24] studied the meanfield social solution to consensus problems. In the basic mean-field decision model, all agents have comparably small influence. However, in some real models, there exists an agent that has a significant influence on other agents. Thus a modified framework is to introduce a major agent interacting

* Corresponding author

1 Zhongtai Securities Institute for Financial Studies, Shandong University, Jinan, Shandong 250100, China. Email: xwfeng@sdu.edu.cn

2 Department of Applied Mathematics, The Hong Kong Polytechnic University, Hong Kong. Email: majhuang@polyu.edu.hk

3 Department of Applied Mathematics, The Hong Kong Polytechnic University, Hong Kong. Email: zhenghong.qiu@connect.polyu.hk with a large number of minor agents. [14] considered LQG major-minor games. [23] studies large population dynamic major-minor games involving nonlinear stochastic dynamical systems. [11] considered heterogeneous major-minor meanfield games. [4] and [5] studied the probabilistic approach to major-minor mean-field games.

In this paper, within the mean-field modeling, we will investigate a new class of stochastic LQG optimization problems involving a major agent and a large number of weakly-coupled minor agents. Specifically, the minor agents are cooperative to minimize the social cost as the sum of individual costs, while the major agent and minor agents are competitive aiming for Nash equilibrium in nonzero-sum game manner.

Our setup is an extension of the well-studied two-player (non-cooperative) game in which two agents make competitive decisions based on individual but centralized information. As an extension, in our setup: one agent no longer applies such centralized decision. Instead, all its sub-units or branches will apply distributed information to optimize the original cost jointly (e.g., [1], [9] and [26]), that now is reformulated in some team-cost form. Thereby, all sub-units become "minor" agents and formalize a (cooperative) team, while another agent still applying centralized information becomes a "major" and non-cooperative player, from the viewpoint of all "minor" agents.

In our study, the problem can be solved in the following way. Firstly, for the major agent, we freeze the state average and obtain the auxiliary control problem. By the result in [29], the auxiliary control problem for the major agent can be derived. Secondly, for the minor agents, under the person-byperson optimality principle, by applying variational techniques and introducing some mean-field terms, the original minor social optimization problem can also be converted to an auxiliary LQG control problem, which can be solved using some traditional scheme in [29] as well. Thirdly, to determine the frozen mean-field terms, we construct the consistency condition (CC) system by some fixed-point analysis. Last, by using some asymptotic analysis and standard estimation of stochastic differential equations (SDEs), we show that the mean-field strategy provides an efficient approximation (i.e., the optimal loss tends to 0 as the population $N$ tends to $\infty$ ).

Moreover, the innovative aspects of the obtained results in this paper are as follows: Firstly, in general mean-field games framework, usually the auxiliary control problem can be obtained directly by replacing the state average with some frozen mean-field term. However, this scheme will bring some 
"bad" strategy in our social optima framework, which can not achieve the asymptotic optimality. Instead, in Section IV, variational techniques are applied to distinguish the highorder infinitesimals after the mean-filed approximation, and a new type of auxiliary control problem would be derived. Secondly, the state process and state average enter the diffusion terms. Such feature brings many difficulties when we apply the variational method to obtain the auxiliary control problem for the minor agents. In particular, $N+1$ additional adjoint processes should be introduced to deal with the cross-terms in the cost functional variation. Thirdly, in the estimation of optimal loss, unlike the general mean-field games framework, the asymptotic optimality is proved through investigating the Fréchet derivative of the social cost in Section VII. Last, the control process enters the diffusion terms. Because of this, the adjoint-state term will enter the drift term of the CC system. This also brings difficulties when we study the solvability of the CC system, which is a mean-field forward-backward stochastic differential equations (MF-FBSDEs) system, and the mean-field terms are represented in an embedding way. To its well-posedness, we apply some discounting methods.

The remaining of the paper is organized as follows: In Section II, we give the formulation of the mixed LQG social optima problem. In Section III and Section IV, we find the auxiliary control problem of the major agent and minor agents respectively. The CC system is derived in Section V. Meanwhile, the well-posedness of CC system is also established. In Section VI, we compare our result with some previous literature. In Section VII, we obtain the asymptotic optimality of the decentralized strategy. Last, in Section VIII, we simulate our model with some numerical methods.

\section{Problem formulation}

Consider a finite time horizon $[0, T]$ for fixed $T>0$. Assume that $\left(\Omega, \mathcal{F},\left\{\mathcal{F}_{t}\right\}_{0 \leq t \leq T}, \mathbb{P}\right)$ is a complete filtered probability space satisfying the usual conditions and $\left\{W_{i}(t), 0 \leq\right.$ $i \leq N\}_{0 \leq t \leq T}$ is an $(N+1)$-dimensional Brownian motion on this space. Let $\mathcal{F}_{t}$ be the filtration generated by $\left\{W_{i}(s), 0 \leq\right.$ $i \leq N\}_{0 \leq s \leq t}$ and augmented by $\mathcal{N}_{\mathbb{P}}$ (the class of all $\mathbb{P}_{-}$ null sets of $\mathcal{F}$ ). Let $\mathcal{F}_{t}^{W_{0}}, \mathcal{F}_{t}^{W_{i}}$ and $\mathcal{F}_{t}^{i}$ be the augmentation of $\sigma\left\{W_{0}(s), 0 \leq s \leq t\right\}, \sigma\left\{W_{i}(s), 0 \leq s \leq t\right\}$ and $\sigma\left\{W_{0}(s), W_{i}(s), 0 \leq s \leq t\right\}$ by $\mathcal{N}_{\mathbb{P}}$ respectively. $\mathbb{E}^{\bar{W}_{0}}$ denotes the conditional expectation w.r.t. $\mathcal{F}^{W_{0}}$.

Let $\langle\cdot, \cdot\rangle$ denote standard Euclidean inner product and $\|\cdot\|$ denote the norm. $x^{\top}$ denotes the transpose of a vector (or matrix) $x . \mathbb{S}^{n}$ denotes the set of symmetric $n \times n$ matrices with real elements. $M>(\geq) 0$ denotes that $M \in \mathbb{S}^{n}$ which is positive (semi)definite, while $M \gg 0$ denotes that, for some $\varepsilon>0, M-\varepsilon I \geq 0$. We introduce the following spaces for any given Euclidean space $\mathbb{H}$. They will be used in the paper:

- $L_{\mathcal{F}_{T}}^{2}(\Omega ; \mathbb{H}):=\left\{\eta: \Omega \rightarrow \mathbb{H} \mid \eta\right.$ is $\mathcal{F}_{T}$-measurable, $\left.\mathbb{E}\|\eta\|^{2}<\infty\right\}$,

- $L_{\mathcal{F}_{t}}^{2}(0, T ; \mathbb{H}):=\left\{\zeta(\cdot):[0, T] \times \Omega \rightarrow \mathbb{H} \mid \zeta(\cdot)\right.$ is $\mathcal{F}_{t^{-}}$ progressively measurable, $\left.\mathbb{E} \int_{0}^{T}\|\zeta(t)\|^{2} d t<\infty\right\}$.

- $L^{\infty}(0, T ; \mathbb{H}):=\left\{\zeta(\cdot):[0, T] \rightarrow \mathbb{H} \mid \operatorname{esssup}_{0 \leq s \leq T}\|\zeta(s)\|<\infty\right\}$.

- $L_{\mathcal{F}_{t}}^{2}(\Omega ; C(0, T ; \mathbb{H})):=\left\{\zeta(\cdot):[0, T] \times \Omega \rightarrow \mathbb{H} \mid \zeta(\cdot)\right.$ is $\mathcal{F}_{t^{-}}$ adapted, continuous, $\left.\mathbb{E}\left(\sup _{0 \leq s \leq T}\|\zeta(s)\|^{2}\right)<\infty\right\}$,

and $\|\zeta\|_{L^{2}}^{2}:=\mathbb{E} \int_{0}^{T}\|\zeta\|^{2} d t$ denotes the $L^{2}$ norm. We consider a weakly coupled large population system with a major agent
$\mathcal{A}_{0}$ and $N$ individual minor agents denoted by $\left\{\mathcal{A}_{i}: 1 \leq i \leq\right.$ $N\}$. The dynamics of the $N+1$ agents are given by a system of SDEs with mean-field coupling:

$$
\left\{\begin{array}{c}
d x_{0}(t)=\left[A_{0}(t) x_{0}(t)+B_{0}(t) u_{0}(t)+F_{0}(t) x^{(N)}(t)\right] d t \\
\quad+\left[C_{0}(t) x_{0}(t)+D_{0}(t) u_{0}(t)+\widetilde{F}_{0}(t) x^{(N)}(t)\right] d W_{0}(t), \\
x_{0}(0)=\xi_{0} \in \mathbb{R}^{n},
\end{array}\right.
$$

and for $1 \leq i \leq N$,

$$
\left\{\begin{array}{l}
d x_{i}(t)=\left[A(t) x_{i}(t)+B(t) u_{i}(t)+F(t) x^{(N)}(t)\right] d t \\
\quad+\left[C(t) x_{i}(t)+D(t) u_{i}(t)+\widetilde{F}(t) x^{(N)}(t)+\widetilde{G}(t) x_{0}(t)\right] d W_{i}(t), \\
x_{i}(0)=\xi \in \mathbb{R}^{n},
\end{array}\right.
$$

where $x^{(N)}(t)=\frac{1}{N} \sum_{i=1}^{N} x_{i}(t)$ is the average state of the minor agents.

Remark 1: We remark that the control process and stateaverage enter both the drift and diffusion terms. This makes our paper different to standard mean-field game (e.g., [28]) or social optimization (e.g., [16]) literature in which only drift terms are control-dependent.

Let $u(\cdot):=\left(u_{0}(\cdot), u_{1}(\cdot), \cdots, u_{N}(\cdot)\right)$ be the set of strategies of all $N+1$ agents, $u_{-0}(\cdot):=\left(u_{1}(\cdot), \cdots, u_{N}(\cdot)\right)$ and $u_{-i}(\cdot):=$ $\left(u_{0}(\cdot), \cdots, u_{i-1}(\cdot), u_{i+1}(\cdot), \cdots, u_{N}(\cdot)\right), 0 \leq i \leq N$. The centralized admissible strategy set is given by

$$
\mathcal{U}_{c}:=\left\{u(\cdot) \mid u(t) \text { is } \mathcal{F}_{t} \text { measurable, } \mathbb{E} \int_{0}^{T}\|u(t)\|^{2} d t<\infty\right\} .
$$

Correspondingly, the feedback decentralized admissible strategy set for the major agent is given by $\mathcal{U}_{0}:=\left\{u_{0}(\cdot) \mid u_{0}(t)\right.$ is $\mathcal{F}_{t}^{W_{0}}$ measurable, $\left.\mathbb{E} \int_{0}^{T}\left\|u_{0}(t)\right\|^{2} d t<\infty\right\}$, and the feedback decentralized admissible strategy set for the $i^{\text {th }}$ minor agent is given by

$\mathcal{U}_{i}:=\left\{u_{i}(\cdot) \mid u_{i}(t)\right.$ is $\mathcal{F}_{t}^{i}$ measurable, $\left.\mathbb{E} \int_{0}^{T}\left\|u_{i}(t)\right\|^{2} d t<\infty\right\}$.

For simplicity, define

$$
\mathcal{U}_{-0}:=\left\{\left(u_{1}(\cdot), \cdots, u_{N}(\cdot)\right) \mid u_{i}(\cdot) \in \mathcal{U}_{i}, i=1, \cdots, N\right\} .
$$

The cost functional for $\mathcal{A}_{0}$ is given by

$$
\begin{aligned}
& \mathcal{J}_{0}\left(u_{0}(\cdot), u_{-0}(\cdot)\right) \\
= & \frac{1}{2} \mathbb{E} \int_{0}^{T}\left[\left\langle Q_{0}(t)\left(x_{0}(t)-H_{0}(t) x^{(N)}(t)\right), x_{0}(t)-H_{0}(t) x^{(N)}(t)\right\rangle\right. \\
& \left.+\left\langle R_{0}(t) u_{0}(t), u_{0}(t)\right\rangle\right] d t,
\end{aligned}
$$

and the cost functional for $\mathcal{A}_{i}, 1 \leq i \leq N$, is given by

$$
\begin{aligned}
& \mathcal{J}_{i}\left(u_{i}(\cdot), u_{-i}(\cdot)\right) \\
= & \frac{1}{2} \mathbb{E} \int_{0}^{T}\left[\left\langleQ(t)\left(x_{i}(t)-H(t) x_{0}(t)-\hat{H}(t) x^{(N)}(t)\right),\right.\right. \\
& \left.\left.x_{i}(t)-H(t) x_{0}(t)-\hat{H}(t) x^{(N)}(t)\right\rangle+\left\langle R(t) u_{i}(t), u_{i}(t)\right\rangle\right] d t .
\end{aligned}
$$

Remark 2: It is worth pointing out that it brings no essential difficulty to introduce a terminal cost term in (3) and (4). This will only change the terminal value of the associated Riccati equations. Thus, for simplicity, we only consider Lagrange type cost functional here.

The aggregate team cost of $N$ minor agents is

$$
\mathcal{J}_{\text {soc }}^{(N)}(u(\cdot))=\sum_{i=1}^{N} \mathcal{J}_{i}\left(u_{i}(\cdot), u_{-i}(\cdot)\right) .
$$

We impose the following general assumptions, which are commonly used in LQG models, on the coefficients:

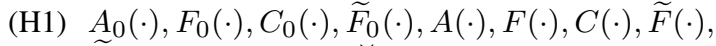
$\widetilde{G}(\cdot) \in L^{\infty}\left(0, T ; \mathbb{R}^{n \times n}\right)$,

$B_{0}(\cdot), D_{0}(\cdot), B(\cdot), D(\cdot) \in L^{\infty}\left(0, T ; \mathbb{R}^{n \times m}\right)$.

(H2) $Q_{0}(\cdot), H_{0}(\cdot), Q(\cdot), H(\cdot), \hat{H}(\cdot) \in L^{\infty}\left(0, T ; \mathbb{S}^{n}\right), R_{0}(\cdot), R(\cdot) \in$ $L^{\infty}\left(0, T ; \mathbb{S}^{m}\right)$. 
Remark 3: Under (H1), the system (1) and (2) admits a unique strong solution $\left(x_{0}, \cdots, x_{N}\right) \in L_{\mathcal{F}_{t}}^{2}\left(\Omega ; C\left(0, T ; \mathbb{R}^{n}\right)\right)$ $\times \cdots \times L_{\mathcal{F}_{t}}^{2}\left(\Omega ; C\left(0, T ; \mathbb{R}^{n}\right)\right)$ for any given admissible control $\left(u_{0}, \cdots, u_{N}\right) \in \mathcal{U}_{c}$. Under (H2), the cost functionals (3) and (4) are well defined.

Note that while the coefficients are dependent on the time variable $t$, in what follows, the variable $t$ will usually be suppressed if no confusion would occur. We propose the following social optimization problem:

Problem 1: Find a decentralized strategy set $\bar{u}(\cdot)=\left(\bar{u}_{0}(\cdot)\right.$, $\left.\bar{u}_{1}(\cdot), \cdots, \bar{u}_{N}(\cdot)\right)$ where $\bar{u}_{i}(\cdot) \in \mathcal{U}_{i}, 0 \leq i \leq N$, such that

$$
\left\{\begin{array}{l}
\mathcal{J}_{0}\left(\bar{u}_{0}(\cdot), \bar{u}_{-0}(\cdot)\right)=\inf _{u_{0} \in \mathcal{U}_{0}} \mathcal{J}_{0}\left(u_{0}(\cdot), \bar{u}_{-0}(\cdot)\right), \\
\mathcal{J}_{\text {soc }}^{(N)}\left(\bar{u}_{0}(\cdot), \bar{u}_{-0}(\cdot)\right)=\inf _{u_{-0} \in \mathcal{U}_{-0}} \mathcal{J}_{\text {soc }}^{(N)}\left(\bar{u}_{0}(\cdot), u_{-0}(\cdot)\right) .
\end{array}\right.
$$

\section{Auxiliary optimal CONTROL PROBLEM OF the MAJOR AGENT}

Replacing $x^{(N)}(\cdot)$ of (1) and (3) by $\hat{x}(\cdot)$ which will be determined in Section V, the limiting major agent's state is given by

$$
\left\{\begin{array}{l}
d z_{0}=\left(A_{0} z_{0}+B_{0} v_{0}+F_{0} \hat{x}\right) d t+\left(C_{0} z_{0}+D_{0} v_{0}+\widetilde{F}_{0} \hat{x}\right) d W_{0}, \\
z_{0}(0)=\xi_{0},
\end{array}\right.
$$

and correspondingly the limiting cost functional is

$$
J_{0}\left(v_{0}(\cdot)\right)=\frac{1}{2} \mathbb{E} \int_{0}^{T}\left[\left\langle Q_{0}\left(z_{0}-H_{0} \hat{x}\right), z_{0}-H_{0} \hat{x}\right\rangle+\left\langle R_{0} v_{0}, v_{0}\right\rangle\right] d t .
$$

We define the following auxiliary stochastic optimal control problem for major agent:

Problem 2: For agent $\mathcal{A}_{0}$, minimize $J_{0}\left(u_{0}(\cdot)\right)$ over $L_{\mathcal{F}_{t}^{W_{0}}}^{2}\left(0, T ; \mathbb{R}^{n}\right)$.

This is a standard LQ stochastic control problem. For its solvability, one can introduce the following standard assumption

(SA) $\quad Q_{0}(\cdot) \geq 0, Q(\cdot) \geq 0, R_{0}(\cdot) \gg 0, R(\cdot) \gg 0$.

By [27, Theorem 4.3], we have the following result:

Proposition 1: Under (H1)-(H2) and (SA), the following Riccati equation

$$
\left\{\begin{array}{l}
-\left(P_{0} B_{0}+C_{0}^{\top} P_{0} D_{0}\right)\left(R_{0}+D_{0}^{\top} P_{0} D_{0}\right)^{-1}\left(B_{0}^{\top} P_{0}+D_{0}^{\top} P_{0} C_{0}\right) \\
+\dot{P}_{0}+P_{0} A_{0}+A_{0}^{\top} P_{0}+C_{0}^{\top} P_{0} C_{0}+Q_{0}=0, \quad P_{0}(T)=0,
\end{array}\right.
$$

is strongly regularly solvable, and Problem 2 admits a feedback optimal control $\bar{v}_{0}=\Theta_{1} \bar{z}_{0}+\Theta_{2}$ where

$$
\left\{\begin{array}{l}
\Theta_{1}=-\left(R_{0}+D_{0}^{\top} P_{0} D_{0}\right)^{-1}\left(B_{0}^{\top} P_{0}+D_{0}^{\top} P_{0} C_{0}\right) \\
\Theta_{2}=-\left(R_{0}+D_{0}^{\top} P_{0} D_{0}\right)^{-1}\left(B_{0}^{\top} \phi+D_{0}^{\top} \zeta+D_{0}^{\top} P_{0} \widetilde{F}_{0} \hat{x}\right)
\end{array}\right.
$$

and $\phi$ satisfies

$$
\left\{\begin{aligned}
d \phi= & -\left\{\left[A_{0}^{\top}-\left(P_{0} B_{0}+C_{0}^{\top} P_{0} D_{0}\right)\left(R_{0}+D_{0}^{\top} P_{0} D_{0}\right)^{-1} B_{0}^{\top}\right] \phi\right. \\
& +\left[C_{0}^{\top}-\left(P_{0} B_{0}+C_{0}^{\top} P_{0} D_{0}\right)\left(R_{0}+D_{0}^{\top} P_{0} D_{0}\right)^{-1} D_{0}^{\top}\right] \zeta \\
& +\left[C_{0}^{\top}-\left(P_{0} B_{0}+C_{0}^{\top} P_{0} D_{0}\right)\left(R_{0}+D_{0}^{\top} P_{0} D_{0}\right)^{-1} D_{0}^{\top}\right] P_{0} \widetilde{F}_{0} \hat{x} \\
& \left.+P_{0} F_{0} \hat{x}-Q_{0} H_{0} \hat{x}\right\} d t+\zeta d W_{0}, \quad \phi(T)=0 .
\end{aligned}\right.
$$

The corresponding optimal state is

$$
\left\{\begin{array}{l}
d \bar{z}_{0}=\left[\left(A_{0}+B_{0} \Theta_{1}\right) \bar{z}_{0}+B_{0} \Theta_{2}+F_{0} \hat{x}\right] d t \\
\quad+\left[\left(C_{0}+D_{0} \Theta_{1}\right) \bar{z}_{0}+D_{0} \Theta_{2}+\widetilde{F}_{0} \hat{x}\right] d W_{0}, \bar{z}_{0}(0)=\xi_{0} .
\end{array}\right.
$$

\section{STOCHASTIC OPTIMAL CONTROL PROBLEM FOR MINOR AGENTS}

\section{A. Person-by-person optimality}

Let $\left(\bar{u}_{1}(\cdot), \cdots, \bar{u}_{n}(\cdot)\right)$ be centralized optimal strategies of the minor agents. We now perturb $u_{i}(\cdot)$ and keep $\bar{u}_{-i}(\cdot)=\left(\bar{u}_{0}(\cdot), \bar{u}_{1}(\cdot), \cdots, \bar{u}_{i-1}(\cdot), \bar{u}_{i+1}(\cdot), \cdots, \bar{u}_{N}(\cdot)\right)$ fixed. For $j=1, \cdots, N, j \neq i$, denote the perturbation $\delta u_{i}(\cdot)=$ $u_{i}(\cdot)-\bar{u}_{i}(\cdot), \delta x_{i}(\cdot)=x_{i}(\cdot)-\bar{x}_{i}(\cdot), \delta x_{j}(\cdot)=x_{j}(\cdot)-\bar{x}_{j}(\cdot)$, $\delta x^{(N)}=\frac{1}{N} \sum_{j=1}^{N} \delta x_{j}(\cdot)$, and $\delta \mathcal{J}_{j}$ is the first variation (Fréchet differential) of $\mathcal{J}_{j}$ w.r.t. $\delta u_{j}$. Therefore, $\delta x_{i}, \delta x_{j}$, $\delta x_{0}$ and $\delta x_{-(0, i)}:=\sum_{j=1, j \neq i}^{N} \delta x_{j}$ are given by

$$
\left\{\begin{aligned}
d \delta x_{i}= & \left(A \delta x_{i}+B \delta u_{i}+F \delta x^{(N)}\right) d t \\
& +\left(C \delta x_{i}+D \delta u_{i}+\widetilde{F} \delta x^{(N)}+\widetilde{G} \delta x_{0}\right) d W_{i}, \\
d \delta x_{j}= & \left(A \delta x_{j}+F \delta x^{(N)}\right) d t+\left(C \delta x_{j}+\widetilde{F} \delta x^{(N)}+\widetilde{G} \delta x_{0}\right) d W_{j}, \\
d \delta x_{0}= & \left(A_{0} \delta x_{0}+F_{0} \delta x^{(N)}\right) d t+\left(C_{0} \delta x_{0}+\widetilde{F}_{0} \delta x^{(N)}\right) d W_{0}, \\
d \delta x_{-(0, i)}= & {\left[A \delta x_{-(0, i)}+F(N-1) \delta x^{(N)}\right] d t } \\
& +\sum_{j \neq i}\left(C \delta x_{j}+\widetilde{F} \delta x^{(N)}+\widetilde{G} \delta x_{0}\right) d W_{j}, \\
\delta x_{i}(0)=0, & \delta x_{j}(0)=0, \delta x_{0}(0)=0, \delta x_{-(0, i)}(0)=0 .
\end{aligned}\right.
$$

By some elementary calculations, we can further obtain $\delta \mathcal{J}_{i}$ of the cost functional of $\mathcal{A}_{i}$ as follows

$\delta \mathcal{J}_{i}=\mathbb{E} \int_{0}^{T}\left\langle Q\left(\bar{x}_{i}-\hat{H} \bar{x}^{(N)}-H \bar{x}_{0}\right), \delta x_{i}-\hat{H} \delta x^{(N)}-H \delta x_{0}\right\rangle+\left\langle R \bar{u}_{i}, \delta u_{i}\right\rangle d t$.

For $j \neq i, \delta \mathcal{J}_{j}$ of the cost functional of $\mathcal{A}_{j}$ is given by

$\delta \mathcal{J}_{j}=\mathbb{E} \int_{0}^{T}\left\langle Q\left(\bar{x}_{j}-\hat{H} \bar{x}^{(N)}-H \bar{x}_{0}\right), \delta x_{j}-\hat{H} \delta x^{(N)}-H \delta x_{0}\right\rangle d t$.

We can further obtain $\delta \mathcal{J}_{\text {soc }}^{(N)}$, the first variation of the social cost, satisfying

$$
\begin{aligned}
& \delta \mathcal{J}_{\text {soc }}^{(N)}=\mathbb{E} \int_{0}^{T}\left[\left\langle Q\left(\bar{x}_{i}-\hat{H} \bar{x}^{(N)}-H \bar{x}_{0}\right), \delta x_{i}-H \delta x_{0}-\hat{H} \delta x^{(N)}\right\rangle\right. \\
& \left.+\sum_{j \neq i}\left\langle Q\left(\bar{x}_{j}-\hat{H} \bar{x}^{(N)}-H \bar{x}_{0}\right), \delta x_{j}-H \delta x_{0}-\hat{H} \delta x^{(N)}\right\rangle+\left\langle R \bar{u}_{i}, \delta u_{i}\right\rangle\right] d t .
\end{aligned}
$$

Replacing $\bar{x}^{(N)}$ in (13) by $\left(\bar{x}^{(N)}-\hat{x}\right)+\hat{x}$,

$$
\begin{gathered}
\delta \mathcal{J}_{\text {soc }}^{(N)}=\mathbb{E} \int_{0}^{T}\left[\left\langle Q \bar{x}_{i}, \delta x_{i}\right\rangle-\left\langle Q\left(\hat{H} \hat{x}+H \bar{x}_{0}\right)+\hat{H} Q\left(\hat{x}-\hat{H} \hat{x}-H \bar{x}_{0}\right), \delta x_{i}\right\rangle\right. \\
-\left\langle\hat{H} Q\left(\hat{x}-\hat{H} \hat{x}-H \bar{x}_{0}\right), \delta x_{-(0, i)}\right\rangle-\left\langle H Q\left(\hat{x}-\hat{H} \hat{x}-H \bar{x}_{0}\right), N \delta x_{0}\right\rangle \\
\left.+\frac{1}{N} \sum_{j \neq i}\left\langle Q\left(\bar{x}_{j}-\hat{H} \hat{x}-H \bar{x}_{0}\right), N \delta x_{j}\right\rangle+\left\langle R \bar{u}_{i}, \delta u_{i}\right\rangle\right] d t+\sum_{l=1}^{4} \varepsilon_{l, i},
\end{gathered}
$$

where

$$
\left\{\begin{array}{l}
\varepsilon_{1, i}=\mathbb{E} \int_{0}^{T}\left\langle(Q \hat{H}-\hat{H} Q \hat{H})\left(\hat{x}-\bar{x}^{(N)}\right), N \delta x^{(N)}\right\rangle d t, \\
\varepsilon_{2, i}=-\mathbb{E} \int_{0}^{T}\left\langle H Q \hat{H}\left(\hat{x}-\bar{x}^{(N)}\right), N \delta x_{0}\right\rangle d t, \\
\varepsilon_{3, i}=\mathbb{E} \int_{0}^{T}\left\langle H Q\left(\hat{x}-\bar{x}^{(N)}\right), N \delta x_{0}\right\rangle d t, \\
\varepsilon_{4, i}=\mathbb{E} \int_{0}^{T}\left\langle\hat{H} Q\left(\hat{x}-\bar{x}^{(N)}\right), N \delta x^{(N)}\right\rangle d t .
\end{array}\right.
$$

Introduce the limit processes $\left(x_{0}^{*}, x_{j}^{*}, x^{* *}\right)$ to replace $\left(N \delta x_{0}\right.$, $\left.N \delta x_{j}, \delta x_{-(0, i)}\right)$ by $\left(\left(N \delta x_{0}-x_{0}^{*}\right)+x_{0}^{*},\left(N \delta x_{j}-x_{j}^{*}\right)+x_{j}^{*}\right.$, $\left.\left(\delta x_{-(0, i)}-x^{* *}\right)+x^{* *}\right)$ where

$$
\left\{\begin{array}{l}
d x_{0}^{*}=\left(A_{0} x_{0}^{*}+F_{0} \delta x_{i}+F_{0} x^{* *}\right) d t+\left(C_{0} x_{0}^{*}+\widetilde{F}_{0} \delta x_{i}+\widetilde{F}_{0} x^{* *}\right) d W_{0}, \\
d x_{j}^{*}=\left(A x_{j}^{*}+F \delta x_{i}+F x^{* *}\right) d t+\left(C x_{j}^{*}+\widetilde{F} \delta x_{i}+\widetilde{F} x^{* *}+\widetilde{G} x_{0}^{*}\right) d W_{j}, \\
d x^{* *}=\left(A x^{* *}+F \delta x_{i}+F x^{* *}\right) d t, \quad x_{0}^{*}(0)=x_{j}^{*}(0)=x^{* *}(0)=0 .
\end{array}\right.
$$


Therefore,

$$
\begin{aligned}
\delta \mathcal{J}_{\text {soc }}^{(N)}= & \mathbb{E} \int_{0}^{T}\left[\left\langle Q \bar{x}_{i}, \delta x_{i}\right\rangle-\left\langle Q\left(\hat{H} \hat{x}+H \bar{x}_{0}\right)+\hat{H} Q\left(\hat{x}-\hat{H} \hat{x}-H \bar{x}_{0}\right), \delta x_{i}\right\rangle\right. \\
& -\left\langle\hat{H} Q\left(\hat{x}-\hat{H} \hat{x}-H \bar{x}_{0}\right), x^{* *}\right\rangle-\left\langle H Q\left(\hat{x}-\hat{H} \hat{x}-H \bar{x}_{0}\right), x_{0}^{*}\right\rangle \\
& \left.+\frac{1}{N} \sum_{j \neq i}\left\langle Q\left(\bar{x}_{j}-\hat{H} \hat{x}-H \bar{x}_{0}\right), x_{j}^{*}\right\rangle+\left\langle R \bar{u}_{i}, \delta u_{i}\right\rangle\right] d t+\sum_{l=1}^{7} \varepsilon_{1, i},
\end{aligned}
$$

where

$$
\left\{\begin{array}{l}
\varepsilon_{5, i}=\mathbb{E} \int_{0}^{T}\left\langle\hat{H} Q\left(\hat{x}-\hat{H} \hat{x}-H \bar{x}_{0}\right), x^{* *}-\delta x_{-(0, i)}\right\rangle d t, \\
\varepsilon_{6, i}=\mathbb{E} \int_{0}^{T}\left\langle H Q\left(\hat{x}-\hat{H} \hat{x}-H \bar{x}_{0}\right), x_{0}^{*}-N \delta x_{0}\right\rangle d t, \\
\varepsilon_{7, i}=\mathbb{E} \int_{0}^{T} \frac{1}{N} \sum_{j \neq i}\left\langle Q\left(\bar{x}_{j}-\hat{H} \hat{x}-H \bar{x}_{0}\right), N \delta x_{j}-x_{j}^{*}\right\rangle d t .
\end{array}\right.
$$

Replacing $\bar{x}_{0}$ by $\left(\bar{x}_{0}-z_{0}\right)+z_{0}$, we have

$$
\begin{aligned}
\delta \mathcal{J}_{\text {soc }}^{(N)}=\mathbb{E} \int_{0}^{T} & {\left[\left\langle Q \bar{x}_{i}, \delta x_{i}\right\rangle-\left\langle Q\left(\hat{H} \hat{x}+H z_{0}\right)+\hat{H} Q(\hat{x}-\hat{H} \hat{x}\right.\right.} \\
& \left.\left.-H z_{0}\right), \delta x_{i}\right\rangle-\left\langle\hat{H} Q\left(\hat{x}-\hat{H} \hat{x}-H z_{0}\right), x^{* *}\right\rangle \\
& -\left\langle H Q\left(\hat{x}-\hat{H} \hat{x}-H z_{0}\right), x_{0}^{*}\right\rangle+\frac{1}{N} \sum_{j \neq i}\left\langleQ \left(\bar{x}_{j}\right.\right. \\
& \left.\left.\left.-\hat{H} \hat{x}-H z_{0}\right), x_{j}^{*}\right\rangle+\left\langle R \bar{u}_{i}, \delta u_{i}\right\rangle\right] d t+\sum_{l=1}^{10} \varepsilon_{l, i},
\end{aligned}
$$

where

$$
\left\{\begin{aligned}
\varepsilon_{8, i}= & \mathbb{E} \int_{0}^{T}\left\langle\hat{H} Q H\left(\bar{x}_{0}-z_{0}\right), x^{* *}\right\rangle d t, \\
& +\mathbb{E} \int_{0}^{T}\left\langle Q H\left(z_{0}-\bar{x}_{0}\right)+\hat{H} Q H\left(z_{0}-\bar{x}_{0}\right), \delta x_{i}\right\rangle d t, \\
\varepsilon_{9, i}= & \mathbb{E} \int_{0}^{T}\left\langle H Q H\left(\bar{x}_{0}-z_{0}\right), x_{0}^{*}\right\rangle d t, \\
\varepsilon_{10, i}= & \mathbb{E} \int_{0}^{T} \frac{1}{N} \sum_{j \neq i}\left\langle Q H\left(z_{0}-\bar{x}_{0}\right), x_{j}^{*}\right\rangle d t .
\end{aligned}\right.
$$

Now we introduce the adjoint equations $y_{1}^{0}, y_{1}^{j}$ and $y_{2}$

$$
\left\{\begin{aligned}
d y_{1}^{0}=[ & H Q\left(\hat{x}-\hat{H} \hat{x}-H z_{0}\right)-A_{0}^{\top} y_{1}^{0}-C_{0}^{\top} \beta_{1}^{0} \\
& -\widetilde{G}^{\top} \mathbb{E}^{W_{0}}\left[\beta_{1}^{j j}\right] d t+\beta_{1}^{0} d W_{0}, \quad y_{1}^{0}(T)=0, \\
d y_{1}^{j}=[ & \left.-Q\left(\bar{x}_{j}-\hat{H} \hat{x}-H z_{0}\right)-A^{\top} y_{1}^{j}-C^{\top} \beta_{1}^{j j}\right] d t \\
& +\beta_{1}^{j j} d W_{j}+\sum_{k \neq j} \beta_{1}^{j k} d W_{k}, \quad y_{1}^{j}(T)=0, \quad j=1, \cdots, N, \\
d y_{2}=[ & \hat{H} Q\left(\hat{x}-\hat{H} \hat{x}-H z_{0}\right)-F^{\top} \mathbb{E}^{W_{0}}\left[y_{1}^{j}\right]-\widetilde{F}^{\top} \mathbb{E}^{W_{0}}\left[\beta_{1}^{j j}\right] \\
& \left.-\widetilde{F}^{\top} \mathbb{E}^{W_{0}}\left[\beta_{1}^{j j}\right]-(A+F)^{\top} y_{2}-F_{0}^{\top} y_{1}^{0}-\widetilde{F}_{0}^{\top} \beta_{1}^{0}\right] d t \\
& +\beta_{2}^{0} d W_{0}, \quad y_{2}(T)=0,
\end{aligned}\right.
$$

to replace the terms $x_{0}^{*}, x_{j}^{*}$ and $x^{* *}$ respectively, where $\beta_{1}^{0}$, $\left(\beta_{1}^{j 1}, \cdots, \beta_{1}^{j N}\right), \beta_{2}^{0}$ are the adjoint-states of $y_{1}^{0}, y_{1}^{j}$ and $y_{2}$ respectively.

Remark 4: It is important to construct an auxiliary LQG control problem for investigating decentralized control in social optima problem. Since (17), the direct variation decomposition of $\delta \mathcal{J}_{\text {soc }}^{(N)}$, contains $x^{* *}, x_{j}^{*}$ and $x_{0}^{*}$, which are some intermediate variation terms related to the basic variation term $\delta x_{i}$ indirectly, we have to use a duality procedure (see [12]) to break away $\delta \mathcal{J}_{\text {soc }}^{(N)}$ from the dependence on $x^{* *}, x_{j}^{*}$ and $x_{0}^{*}$. To this end, we introduce such three adjoint equations.
Applying Itô's formula to $\left\langle y_{1}^{j}, x_{j}^{*}\right\rangle,\left\langle y_{2}, x^{* *}\right\rangle$ and $\left\langle y_{1}^{0}, x_{0}^{*}\right\rangle$, we have

$$
\begin{aligned}
& 0=\mathbb{E}\left\langle y_{1}^{j}(T), x_{j}^{*}(T)\right\rangle-\mathbb{E}\left\langle y_{1}^{j}(0), x_{j}^{*}(0)\right\rangle \\
& =\mathbb{E} \int_{0}^{T}\left[\left\langle-Q\left(\bar{x}_{j}-\hat{H} \hat{x}-H z_{0}\right), x_{j}^{*}\right\rangle+\left\langle F^{\top} y_{1}^{j}+\widetilde{F} \beta_{1}^{j j}, x^{* *}\right\rangle\right. \\
& \left.+\left\langle\widetilde{G}^{\top} \beta_{1}^{j j}, x_{0}^{*}\right\rangle+\left\langle F^{\top} y_{1}^{j}+\widetilde{F}^{\top} \beta_{1}^{j j}, \delta x_{i}\right\rangle\right] d t . \\
& =\mathbb{E}\left\langle y_{2}(T), x^{* *}(T)\right\rangle-\mathbb{E}\left\langle y_{2}(0), x^{* *}(0)\right\rangle \\
& =\mathbb{E} \int_{0}^{T}\left[\left\langle\hat{H} Q\left(\hat{x}-\hat{H} \hat{x}-H z_{0}\right)-F^{\top} \mathbb{E}^{W_{0}}\left[y_{1}^{j}\right]\right.\right. \\
& \left.\left.-\widetilde{F}^{\top} \mathbb{E}^{W_{0}}\left[\beta_{1}^{j j}\right]-F_{0}^{\top} y_{1}^{0}-\widetilde{F}_{0}^{\top} \beta_{1}^{0}, x^{* *}\right\rangle+\left\langle F^{\top} y_{2}, \delta x_{i}\right\rangle\right] d t, \\
& 0=\mathbb{E}\left\langle y_{1}^{0}(T), x_{0}^{*}(T)\right\rangle-\mathbb{E}\left\langle y_{1}^{0}(0), x_{0}^{*}(0)\right\rangle \\
& =\mathbb{E} \int_{0}^{T}\left[\left\langle H Q\left(\hat{x}-\hat{H} \hat{x}-H z_{0}\right)-\widetilde{G}^{\top} \mathbb{E}^{W_{0}}\left[\beta_{1}^{j j}\right], x_{0}^{*}\right\rangle\right. \\
& \left.+\left\langle F_{0}^{\top} y_{1}^{0}+\widetilde{F}_{0}^{\top} \beta_{1}^{0}, x^{* *}\right\rangle+\left\langle F_{0}^{\top} y_{1}^{0}+\widetilde{F}_{0}^{\top} \beta_{1}^{0}, \delta x_{i}\right\rangle\right] d t .
\end{aligned}
$$

Adding to (17), we have

$$
\begin{aligned}
& \delta \mathcal{J}_{\text {soc }}^{(N)}=\mathbb{E} \int_{0}^{T}\left[\left\langle Q \bar{x}_{i}, \delta x_{i}\right\rangle-\left\langle Q\left(\hat{H} \hat{x}+H z_{0}\right)+\hat{H} Q\left(\hat{x}-\hat{H} \hat{x}-H z_{0}\right)\right.\right. \\
& -F^{\top} y_{2}-F^{\top} \mathbb{E}\left[y_{1}^{j} \mid \mathcal{F}_{t}^{W_{0}}\right]-\widetilde{F}^{\top} \mathbb{E}\left[\beta_{1}^{j j} \mid \mathcal{F}_{t}^{W_{0}}\right]-F_{0}^{\top} y_{1}^{0} \\
& \left.\left.-\widetilde{F}_{0}^{\top} \beta_{1}^{0}, \delta x_{i}\right\rangle+\left\langle R \bar{u}_{i}, \delta u_{i}\right\rangle\right] d t+\sum_{l=1}^{13} \varepsilon_{l},
\end{aligned}
$$

where

$$
\left\{\begin{aligned}
& \varepsilon_{11, i}=\mathbb{E} \int_{0}^{T}\left\langle F^{\top}\right.\left(\frac{1}{N} \sum_{j \neq i} y_{1}^{j}-\mathbb{E}^{W_{0}}\left[y_{1}^{j}\right]\right) \\
&\left.+\widetilde{F}^{\top}\left(\frac{1}{N} \sum_{j \neq i} \beta_{1}^{j j}-\mathbb{E}^{W_{0}}\left[\beta_{1}^{j j}\right]\right), x^{* *}\right\rangle d t, \\
& \varepsilon_{12, i}=\mathbb{E} \int_{0}^{T}\left\langle\widetilde{G}^{\top}\left(\frac{1}{N} \sum_{j \neq i} \beta_{1}^{j j}-\mathbb{E}^{W_{0}}\left[\beta_{1}^{j j}\right]\right), x_{0}^{*}\right\rangle d t, \\
& \varepsilon_{13, i}=\mathbb{E} \int_{0}^{T}\left\langle F^{\top}\left(\frac{1}{N} \sum_{j \neq i} y_{1}^{j}-\mathbb{E}^{W_{0}}\left[y_{1}^{j}\right]\right)\right. \\
&\left.+\widetilde{F}^{\top}\left(\frac{1}{N} \sum_{j \neq i} \beta_{1}^{j j}-\mathbb{E}^{W_{0}}\left[\beta_{1}^{j j}\right]\right), \delta x_{i}\right\rangle d t .
\end{aligned}\right.
$$

Therefore, considering the case when $N \longrightarrow \infty$, we introduce the first variation of the decentralized auxiliary cost functional $\delta J_{i}$ as follows

$$
\begin{aligned}
& \delta J_{i}=\mathbb{E} \int_{0}^{T}\left[\left\langle Q \bar{x}_{i}, \delta x_{i}\right\rangle-\left\langle Q\left(\hat{H} \hat{x}+H z_{0}\right)+\hat{H} Q\left(\hat{x}-\hat{H} \hat{x}-H z_{0}\right)\right.\right. \\
& \left.\left.-F^{\top} y_{2}-F^{\top} \hat{y}_{1}-\widetilde{F}^{\top} \hat{\beta}_{1}-F_{0}^{\top} y_{1}^{0}-\widetilde{F}_{0}^{\top} \beta_{1}^{0}, \delta x_{i}\right\rangle+\left\langle R \bar{u}_{i}, \delta u_{i}\right\rangle\right] d t .
\end{aligned}
$$

Remark 5: In (23), we ignore $\varepsilon_{1}, \cdots, \varepsilon_{13}$ and introduce the first variation of the auxiliary cost functional $\delta J_{i}$. Actually, $\varepsilon_{1}, \cdots, \varepsilon_{13}$ have some order as $\bar{x}^{(N)}-\hat{x}$, and it is sufficient to conjecture $\left\|\bar{x}^{(N)}-\hat{x}\right\|_{L^{2}}^{2} \longrightarrow 0$ when $N \longrightarrow \infty$ by considering the weakly coupled structure of our problem. The rigorous proof will be given in Section VII.

\section{B. Decentralized strategy}

Motivated by (23), one can introduce the following auxiliary problem:

Problem 3: Minimize $J_{i}\left(u_{i}\right)$ over $u_{i} \in \mathcal{U}_{i}$ where

$$
\left\{\begin{aligned}
d z_{i}= & \left(A z_{i}+B v_{i}+F \hat{x}\right) d t \\
& +\left(C z_{i}+D v_{i}+\widetilde{F} \hat{x}+\widetilde{G} z_{0}\right) d W_{i}, \quad z_{i}(0)=z, \\
J_{i}\left(v_{i}\right)= & \frac{1}{2} \mathbb{E} \int_{0}^{T}\left[\left\langle Q z_{i}, z_{i}\right\rangle-2\left\langle S, z_{i}\right\rangle+\left\langle R v_{i}, v_{i}\right\rangle\right] d t \\
S= & Q\left(\hat{H} \hat{x}+H z_{0}\right)+\hat{H} Q\left(\hat{x}-\hat{H} \hat{x}-H z_{0}\right)-F^{\top} y_{2} \\
& -F^{\top} \hat{y}_{1}-\widetilde{F}^{\top} \hat{\beta}_{1}-F_{0}^{\top} y_{1}^{0}-\widetilde{F}_{0}^{\top} \beta_{1}^{0} .
\end{aligned}\right.
$$


The mean-field terms $\hat{x}, z_{0}, y_{2}, \hat{y}_{1}, \hat{\beta}_{1}, y_{1}^{0}, \beta_{1}^{0}$ will be determined by the CC system in Section V. From [27], we have the following result:

Proposition 2: Under (H1)-(H2) and (SA), the following Riccati equation

$$
\left\{\begin{array}{l}
\dot{P}+P A+A^{\top} P+C^{\top} P C+Q-\left(P B+C^{\top} P D\right) \\
\times\left(R+D^{\top} P D\right)^{-1}\left(B^{\top} P+D^{\top} P C\right)=0, \quad P(T)=0,
\end{array}\right.
$$

is strongly regularly solvable, and Problem 3 admits a feedback optimal control $\bar{v}_{i}=\Lambda_{1} \bar{x}_{i}+\Lambda_{2}$ where

$$
\left\{\begin{array}{l}
\Lambda_{1}=-\left(R+D^{\top} P D\right)^{-1}\left(B^{\top} P+D^{\top} P C\right) \\
\Lambda_{2}=-\left(R+D^{\top} P D\right)^{-1}\left(B^{\top} \varphi+D^{\top} \eta+D^{\top} P\left(\widetilde{F} \hat{x}+\widetilde{G} z_{0}\right)\right),
\end{array}\right.
$$

and $(\varphi, \eta)$ satisfies

$$
\left\{\begin{aligned}
d \varphi= & -\left\{\left[A^{\top}-\left(P B+C^{\top} P D\right)\left(R+D^{\top} P D\right)^{-1} B^{\top}\right] \varphi\right. \\
& +\left[C^{\top}-\left(P B+C^{\top} P D\right)\left(R+D^{\top} P D\right)^{-1} D^{\top}\right] \eta \\
& +\left[\left(P B+C^{\top} P D\right)\left(R+D^{\top} P D\right)^{-1} D^{\top}-C^{\top}\right] \\
& \left.\times P\left(\widetilde{F} \hat{x}+\widetilde{G} z_{0}\right)+P F \hat{x}-S\right\} d t+\eta d W_{0}, \varphi(T)=0 .
\end{aligned}\right.
$$

\section{CONSISTENCY CONDITION}

Because of the symmetric and decentralized character, we only need a generic Brownian motion (still denoted by $W_{1}$ ) which is independent of $W_{0}$ to characterize the CC system.

Proposition 3: The undetermined quantities in Problem 2 3 can be determined by $\left(\hat{x}, z_{0}, y_{1}^{0}, \beta_{1}^{0}, \hat{y}_{1}, \hat{\beta}_{1}, y_{2}\right)=\left(\mathbb{E}^{W_{0}}[z]\right.$, $\left.z_{0}, \check{y}_{0}, \check{\beta}_{0}, \mathbb{E}^{W_{0}}\left[\check{y}_{1}\right], \mathbb{E}^{W_{0}}\left[\check{\beta}_{1}^{1}\right], \check{y}_{2}\right)$, where $\left(z, z_{0}, \check{y}_{0}, \check{\beta}_{0}, \check{y}_{1}\right.$, $\left.\check{\beta}_{1}^{1}, \breve{y}_{2}\right)$ is the solution of the following MF-FBSDEs:

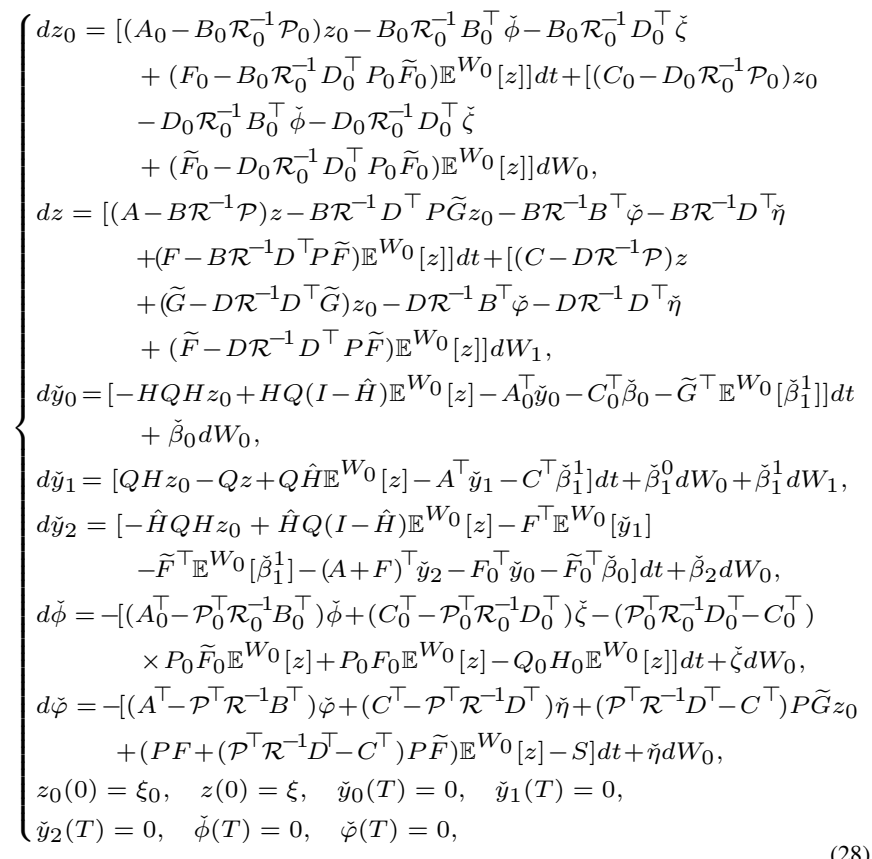

with

$$
\left\{\begin{array}{l}
\mathcal{P}:=B^{\top} P+D^{\top} P C, \quad \mathcal{P}_{0}:=B_{0}^{\top} P_{0}+D_{0}^{\top} P_{0} C_{0} \\
\mathcal{R}:=R+D^{\top} P D, \quad \mathcal{R}_{0}:=R_{0}+D_{0}^{\top} P_{0} D_{0}
\end{array}\right.
$$

Define $X=\left(z_{0}^{\top}, z^{\top}\right)^{\top}, Y=\left(\check{y}_{0}^{\top}, \check{y}_{1}^{\top}, \check{y}_{2}^{\top}, \check{\phi}^{\top}, \check{\varphi}^{\top}\right)^{\top}, Z_{1}=\left(\check{\beta}_{0}^{\top}, \check{\beta}_{1}^{0 \top}\right.$, $\left.\check{\beta}_{2}^{\top}, \check{\zeta}^{\top}, \check{\eta}^{\top}\right)^{\top}, Z_{2}=\left(0^{\top}, \check{\beta}_{1}^{1 \top}, 0^{\top}, 0^{\top}, 0^{\top}\right)^{\top}, Z=\left(Z_{1}, Z_{2}\right)$ and $W=$
$\left(W_{0}^{\top}, W_{1}^{\top}\right)^{\top},(28)$ take the following form:

$$
\left\{\begin{aligned}
d X= & {\left[\mathbb{A}_{1} X+\overline{\mathbb{A}}_{1} \mathbb{E}\left[X \mid \mathcal{F}_{t}^{W_{0}}\right]+\mathbb{B}_{1} Y+\mathbb{F}_{1} Z_{1}\right] d t } \\
& +\left[\mathbb{C}_{1}^{0} X+\overline{\mathbb{C}}_{1}^{0} \mathbb{E}\left[X \mid \mathcal{F}_{t}^{W_{0}}\right]+\mathbb{D}_{1}^{0} Y+\mathbb{F}_{1}^{0} Z_{1}\right] d W_{0} \\
& +\left[\mathbb{C}_{1}^{1} X+\overline{\mathbb{C}}_{1}^{1} \mathbb{E}\left[X \mid \mathcal{F}_{t}^{W_{0}}\right]+\mathbb{D}_{1}^{1} Y+\mathbb{F}_{1}^{1} Z_{1}\right] d W_{1}, \\
d Y= & {\left[\mathbb{A}_{2} X+\overline{\mathbb{A}}_{2} \mathbb{E}\left[X \mid \mathcal{F}_{t}^{W_{0}}\right]+\mathbb{B}_{2} Y+\overline{\mathbb{B}}_{2} \mathbb{E}\left[Y \mid \mathcal{F}_{t}^{W_{0}}\right]\right.} \\
& \left.+\mathbb{C}_{2} Z_{1}+\widetilde{\mathbb{C}}_{2} Z_{2}+\overline{\mathbb{C}}_{2} \mathbb{E}\left[Z_{2} \mid \mathcal{F}_{t}^{W_{0}}\right]\right] d t+Z_{1} d W_{0}+Z_{2} d W_{1}, \\
X(0)= & \left(\xi_{0}^{\top}, \xi^{\top}\right)^{\top}, \quad Y(T)=\left(0^{\top}, 0^{\top}, 0^{\top}, 0^{\top}, 0^{\top}\right)^{\top},
\end{aligned}\right.
$$

where

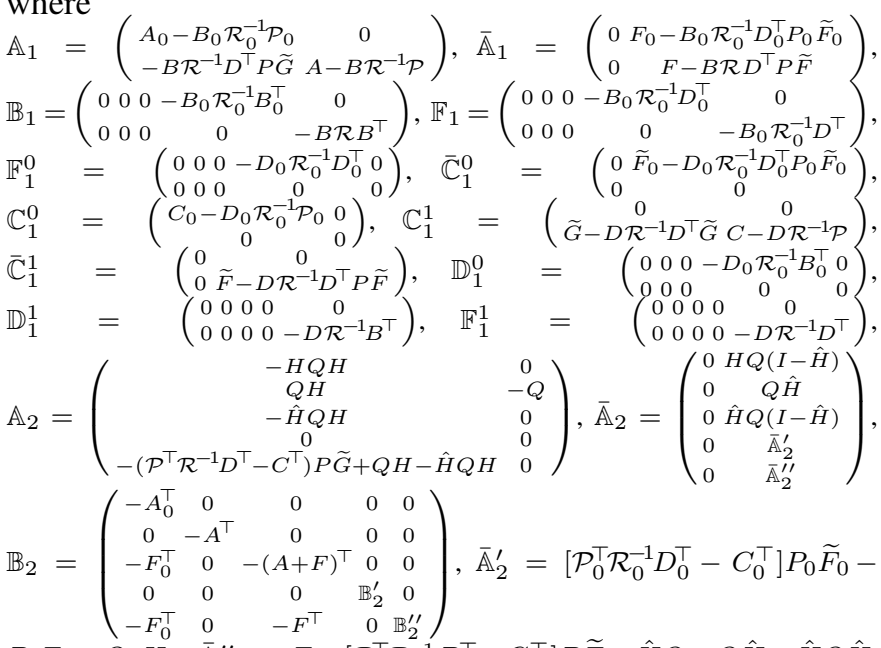
$P_{0} F_{0}+Q_{0} H_{0}, \overline{\mathbb{A}}_{2}^{\prime \prime}=-F+\left[\mathcal{P}^{\top} \mathcal{R}^{-1} D^{\top}-C^{\top}\right] P \widetilde{F}+\hat{H} Q+Q \hat{H}-\hat{H} Q \hat{H}$, $\mathbb{B}_{2}^{\prime}=-A_{0}^{\top}+\mathcal{P}_{0}^{\top} \mathcal{R}_{0}^{-1} B_{0}^{\top}, \mathbb{B}_{2}^{\prime \prime}=-A^{\top}+\mathcal{P}^{\top} \mathcal{R}^{-1} B^{\top}, \bar{B}_{2}=\left(\begin{array}{ccccc}0 & 0 & 0 & 0 & 0 \\ 0 & 0 & 0 & 0 & 0 \\ 0 & -F^{\top} & 0 & 0 & 0 \\ 0 & 0 & 0 & 0 & 0 \\ 0 & -F^{\top} & 0 & 0 & 0\end{array}\right)$, $\overline{\mathbb{C}}_{2}=\left(\begin{array}{ccccc}0 & -\widetilde{G}^{\top} & 0 & 0 & 0 \\ 0 & 0 & 0 & 0 & 0 \\ 0 & -\widetilde{F}^{\top} & 0 & 0 & 0 \\ 0 & 0 & 0 & 0 & 0 \\ 0 & -\widetilde{F}^{\top} & 0 & 0 & 0\end{array}\right), \mathbb{C}_{2}^{\prime}=-\left(C_{0}^{\top}-\mathcal{P}_{0}^{\top} \mathcal{R}_{0}^{-1} D_{0}^{\top}\right), \mathbb{C}_{2}^{\prime \prime}=-\left(C^{\top}-\mathcal{P}^{\top} \mathcal{R}^{-1} D^{\top}\right)$. Next we use discounting method to study the global solvability of FBSDEs (30). To start, we first give some results for general nonlinear forward-backward system:

$$
\left\{\begin{aligned}
& d X(t)= b\left(t, X(t), \mathbb{E}^{W_{0}}[X(t)], Y(t), Z(t)\right) d t \\
&+\sigma\left(t, X(t), \mathbb{E}^{W_{0}}[X(t)], Y(t), Z(t)\right) d W(t), \\
& d Y(t)=-f\left(t, X(t), \mathbb{E}^{W_{0}}[X(t)], Y(t), \mathbb{E}\left[Y(t) \mid \mathcal{F}_{t}^{W_{t}}\right],\right. \\
&\left.Z(t), \mathbb{E}^{W_{0}}[Z(t)]\right) d t+Z(t) d W(t), \\
& X(0)=x, \quad Y(T)=0,
\end{aligned}\right.
$$

where $W=\left(\begin{array}{c}W_{0} \\ W_{1}\end{array}\right)$, and the coefficients satisfy the following conditions:

(A1) There exist $\rho_{1}, \rho_{2} \in \mathbb{R}$ and positive constants $k_{i}, i=$ $1, \cdots, 12$ such that for all $t, x, \bar{x}, y, \bar{y}, z, \bar{z}$, a.s., leftmargin $=^{*}$

1) $\left\langle b\left(t, x_{1}, \bar{x}, y, z\right)-b\left(t, x_{2}, \bar{x}, y, z\right), x_{1}-x_{2}\right\rangle \leq$ $\rho_{1}\left\|x_{1}-x_{2}\right\|^{2}$

2) $\left\|b\left(t, x, \bar{x}_{1}, y_{1}, z_{1}\right)-b\left(t, x, \bar{x}_{2}, y_{2}, z_{2}\right)\right\| \leq k_{1} \| \bar{x}_{1}-$ $\bar{x}_{2}\left\|+k_{2}\right\| y_{1}-y_{2}\left\|+k_{3}\right\| z_{1}-z_{2} \|$

3) $\left\langle f\left(t, x, \bar{x}, y_{1}, \bar{y}, z, \bar{z}\right)-f\left(t, x, \bar{x}, y_{2}, \bar{y}, z, \bar{z}\right)\right.$, $\left.y_{1}-y_{2}\right\rangle \leq \rho_{2}\left\|y_{1}-y_{2}\right\|^{2}$

4) $\| f\left(t, x_{1}, \bar{x}_{1}, y, \bar{y}_{1}, z_{1}, \bar{z}_{1}\right)-f\left(t, x_{2}, \bar{x}_{2}, y, \bar{y}_{2}, z_{2}\right.$, $\left.\bar{z}_{2}\right)\left\|\leq k_{4}\right\| x_{1}-x_{2}\left\|+k_{5}\right\| \bar{x}_{1}-\bar{x}_{2}\left\|+k_{6}\right\| \bar{y}_{1}-\bar{y}_{2} \|$ $+k_{7}\left\|z_{1}-z_{2}\right\|+k_{8}\left\|\bar{z}_{1}-\bar{z}_{2}\right\|$,

5) $\left\|\sigma\left(t, x_{1}, \bar{x}_{1}, y_{1}, z_{1}\right)-\sigma\left(t, x_{2}, \bar{x}_{2}, y_{2}, z_{2}\right)\right\|^{2} \leq$ $k_{9}^{2}\left\|x_{1}-x_{2}\right\|^{2}+k_{10}^{2}\left\|\bar{x}_{1}-\bar{x}_{2}\right\|^{2}+k_{11}^{2}\left\|y_{1}-y_{2}\right\|^{2}+$ $k_{12}^{2}\left\|z_{1}-z_{2}\right\|^{2}$. 
(A2)

$$
\mathbb{E} \int_{0}^{T}\|b(t, 0,0,0,0)\|^{2}+\|\sigma(t, 0,0,0,0)\|^{2}+\|f(t, 0,0,0,0,0,0)\|^{2} d t<\infty .
$$

Similar to [11], we have the following result of solvability of (30). For the readers' convenience, we give the proof in the appendix.

Theorem 1: Suppose (A1) and (A2) hold. There exists a constant $\delta_{1}>0$ depending on $\rho_{1}, \rho_{2}, T, k_{i}, i=1,6,7,8,9$, 10 such that if $k_{i} \in\left[0, \delta_{1}\right), i=2,3,4,5,11,12$, FBSDE (31) admits a unique adapted solution $(X, Y, Z) \in L_{\mathcal{F}}^{2}\left(0, T ; \mathbb{R}^{n}\right) \times$ $L_{\mathcal{F}}^{2}\left(0, T ; \mathbb{R}^{m}\right) \times L_{\mathcal{F}}^{2}\left(0, T ; \mathbb{R}^{m \times d}\right)$. Furthermore, if $2 \rho_{1}+2 \rho_{2}<$ $-2 k_{1}-2 k_{6}-2 k_{7}^{2}-2 k_{8}^{2}-k_{9}^{2}-k_{10}^{2}$, there exists a constant $\delta_{2}>0$ depending on $\rho_{1}, \rho_{2}, k_{i}, i=1,6,7,8,9,10$ such that if $k_{i} \in\left[0, \delta_{2}\right), i=2,3,4,5,11,12$, FBSDE (31) admits a unique adapted solution $(X, Y, Z) \in L_{\mathcal{F}}^{2}\left(0, T ; \mathbb{R}^{n}\right) \times L_{\mathcal{F}}^{2}\left(0, T ; \mathbb{R}^{m}\right) \times$ $L_{\mathcal{F}}^{2}\left(0, T ; \mathbb{R}^{m \times d}\right)$.

Let $\rho_{1}^{*}$ and $\rho_{2}^{*}$ be the largest eigenvalue of $\frac{1}{2}\left(\mathbb{A}_{1}+\mathbb{A}_{1}^{\top}\right)$ and $\frac{1}{2}\left(\mathbb{B}_{2}+\mathbb{B}_{2}^{\top}\right)$ respectively. Comparing (31) with (30), we can check that the parameters of (A1) can be chosen as follows:

$k_{1}=\left\|\overline{\mathbb{A}}_{1}\right\|, \quad k_{2}=\left\|\mathbb{B}_{1}\right\|, \quad k_{3}=\left\|F_{1}\right\|, \quad k_{4}=\left\|\mathbb{A}_{2}\right\|, \quad k_{5}=\left\|\overline{\mathbb{A}}_{2}\right\|$, $k_{6}=\left\|\overline{\mathbb{B}}_{2}\right\|, \quad k_{7}=\left\|\mathbb{C}_{2}\right\|+\left\|\tilde{\mathbb{C}}_{2}\right\|, \quad k_{8}=\left\|\overline{\mathbb{C}}_{2}\right\|, \quad k_{9}=\left\|\mathbb{C}_{1}^{0}\right\|+\left\|\mathbb{C}_{1}^{1}\right\|$, $k_{10}=\left\|\overline{\mathbb{C}}_{1}^{0}\right\|+\left\|\overline{\mathbb{C}}_{1}^{1}\right\|, \quad k_{11}=\left\|\mathbb{D}_{1}^{0}\right\|+\left\|\mathbb{D}_{1}^{1}\right\|, \quad k_{12}=\left\|F_{1}^{0}\right\|+\left\|F_{1}^{1}\right\|$.

Now we introduce the following assumption:

(H3) $\quad 2 \rho_{1}^{*}+2 \rho_{2}^{*}<-2\left\|\overline{\mathbb{A}}_{1}\right\|-2\left\|\overline{\mathbb{B}}_{2}\right\|-2\left(\left\|\mathbb{C}_{2}\right\|+\left\|\tilde{\mathbb{C}}_{2}\right\|\right)^{2}-$ $2\left\|\overline{\mathbb{C}}_{2}\right\|^{2}-\left(\left\|\mathbb{C}_{1}^{0}\right\|+\left\|\mathbb{C}_{1}^{1}\right\|\right)^{2}-\left(\left\|\overline{\mathbb{C}}_{1}^{0}\right\|+\left\|\overline{\mathbb{C}}_{1}^{1}\right\|\right)^{2}$.

We have the following result:

Proposition 4: Under (H1)-(H3), there exists a constant $\delta_{3}>0$ depending on $\rho_{1}^{*}, \rho_{2}^{*}, k_{i}, i=1,6,7,8,9,10$, such that if $k_{i} \in\left[0, \delta_{3}\right), i=2,3,4,5,11,12$, FBSDEs (30) admits a unique adapted solution $(X, Y, Z) \in L_{\mathcal{F}}^{2}\left(0, T ; \mathbb{R}^{n}\right) \times$ $L_{\mathcal{F}}^{2}\left(0, T ; \mathbb{R}^{m}\right) \times L_{\mathcal{F}}^{2}\left(0, T ; \mathbb{R}^{m \times d}\right)$.

In what follows, we give an example to show how exactly such conditions can be applied.

Remark 6: For $\varepsilon>0$, let $\rho_{1}=\frac{k_{2}}{\varepsilon}, \rho_{2}=\frac{k_{3}}{\varepsilon}, \rho_{3}=\frac{k_{4}}{\varepsilon}$, $\rho_{4}=\frac{k_{5}}{\varepsilon}, \rho_{5}=\frac{k_{7}}{2 k_{7}^{2}+2 \varepsilon}, \rho_{6}=\frac{k_{8}}{2 k_{8}^{2}+2 \varepsilon}, d=-2 k_{1}-2 k_{6}-2 k_{7}^{2}-$ $2 k_{8}^{2}-k_{9}^{2}-k_{1} 0^{2}-2 \rho_{1}^{*}-2 \rho_{2}^{*}-4 \varepsilon, \bar{\rho}_{1}=\bar{\rho}_{2}=\frac{d}{2}, \theta=\left(\frac{1}{\bar{\rho}_{2}}+\right.$ $\left.\frac{1}{1-k_{7} \rho_{5}-k_{8} \rho_{10}}\right)\left(\frac{1}{\bar{\rho}_{1}}\right)=\left(\frac{2}{d}+\frac{\left(k_{7}^{2}+\varepsilon\right)\left(k_{8}^{2}+\varepsilon\right)}{\varepsilon^{2}}\right)\left(\frac{2}{d}\right)$. If $d>0, \theta\left(\frac{k_{4}^{2}}{\varepsilon}+\right.$ $\left.\frac{k_{5}^{2}}{\varepsilon}\right)<1, \theta\left(\frac{k_{2}^{2}}{\varepsilon}+k_{11}^{2}\right)<1, \theta\left(\frac{k_{3}^{2}}{\varepsilon}+k_{12}^{2}\right)<1$, then (30) admits a unique solution.

Thus, via Propositions 1, 2, 4, we can establish the following procedure to calculate the mean-field strategy.

- Under (H1)-(H3) and (SA), each agent can calculate CC system (28) and obtain $\left(z, z_{0}, \check{y}_{0}, \check{\beta}_{0}, \check{y}_{1}, \check{\beta}_{1}^{1}, \check{y}_{2}\right)$. Then by taking expectation, the mean-field terms can be obtained by $\left(\hat{x}, z_{0}, y_{1}^{0}, \beta_{1}^{0}, \hat{y}_{1}, \hat{\beta}_{1}, y_{2}\right)=\left(\mathbb{E}^{W_{0}}[z], z_{0}, \check{y}_{0}\right.$, $\left.\check{\beta}_{0}, \mathbb{E}^{W_{0}}\left[\check{y}_{1}\right], \mathbb{E}^{W_{0}}\left[\check{\beta}_{1}^{1}\right], \check{y}_{2}\right)$.

- With $\left(\hat{x}, z_{0}, y_{1}^{0}, \beta_{1}^{0}, \hat{y}_{1}, \hat{\beta}_{1}, y_{2}\right)$, the agent can solve Riccati equations (9), (25) and BSDEs (11), (27) to obtain $P_{0}, P, \phi, \varphi$.

- With $P_{0}, P, \phi, \varphi$, the agent can obtain $\left(\Theta_{1}, \Theta_{2}\right)$ and $\left(\Lambda_{1}, \Lambda_{2}\right)$ by (10) and (26) respectively. Then the meanfield decentralized strategies are given by $\tilde{u}_{0}=\Theta_{1} \bar{z}_{0}+$ $\Theta_{2}, \tilde{u}_{i}=\Lambda_{1} \bar{z}_{i}+\Lambda_{2}$, for $i=1, \cdots, N$, where $\bar{z}_{0}$ and $\bar{z}_{i}$ satisfy

$$
\left\{\begin{aligned}
& d \bar{z}_{0}=\left(\left(A_{0}+B_{0} \Theta_{1}\right) \bar{z}_{0}+B \Theta_{2}+F_{0} \mathbb{E}^{W_{0}}[z]\right) d t \\
&+\left(\left(C_{0}+D_{0} \Theta_{1}\right) \bar{z}_{0}+D_{0} \Theta_{2}+\widetilde{F}_{0} \mathbb{E}^{W_{0}}[z]\right) d W_{0} \\
& d \bar{z}_{i}=\left(\left(A+B \Lambda_{1}\right) \bar{z}_{i}+B \Lambda_{2}+F \mathbb{E}^{W_{0}}[z]\right) d t \\
&+\left(\left(C+D \Lambda_{1}\right) \bar{z}_{i}+D \Lambda_{2}+\widetilde{F} \mathbb{E}^{W_{0}}[z]+\widetilde{G} \bar{z}_{0}\right) d W_{i} \\
& \bar{z}_{0}(0)=\xi_{0}, \quad \bar{z}_{i}(0)=\xi, \quad 1 \leq i \leq N
\end{aligned}\right.
$$

Through the discussion above, the mean-field decentralized strategies are characterized. In what follows, we will show some special cases and illustrate the relation between our research and the existing literature.

\section{Special CASE}

In this section, we compare our result with standard LQG control problem [27] and LQG social optima [25].

When there involves no minor agent, this problem will reduce to a standard LQG optimal control problem. By letting $A=B=F=C=D=\tilde{F}=\tilde{G}=Q=H=\hat{H}=R=0$, we have $x_{i}^{(N)}=x_{i} \equiv 0$, and also by (28), we have $z=\hat{x} \equiv 0$. Then by (9) and (11), we have

$\left\{\begin{array}{l}-\left(P_{0} B_{0}+C_{0}^{\top} P_{0} D_{0}\right)\left(R_{0}+D_{0}^{\top} P_{0} D_{0}\right)^{-1}\left(B_{0}^{\top} P_{0}+D_{0}^{\top} P_{0} C_{0}\right)+ \\ \dot{P}_{0}+P_{0} A_{0}+A_{0}^{\top} P_{0}+C_{0}^{\top} P_{0} C_{0}+Q_{0}=0, \quad P_{0}(T)=0,\end{array}\right.$ and $\phi \equiv 0$. Thus, $\left(\Theta_{1}, \Theta_{2}\right)$ takes the following form

$\Theta_{1}=-\left(R_{0}+D_{0}^{\top} P_{0} D_{0}\right)^{-1}\left(B_{0}^{\top} P_{0}+D_{0}^{\top} P_{0} C_{0}\right), \quad \Theta_{2}=0$.

Such result is consistent with [27, Theorem 4.3].

On the other hand, when there involves no major agent, this problem will reduce to a problem of mean-field control in social optima. By letting $A_{0}=B_{0}=F_{0}=C_{0}=D_{0}=\tilde{F}_{0}$ = $\tilde{G}_{0}=Q_{0}=H_{0}=\hat{H}_{0}=R_{0}=0$, we have $x_{0} \equiv 0$. The CC system becomes:

$$
\left\{\begin{aligned}
& d z= {\left[\left(A-B \mathcal{R}^{-1} \mathcal{P}\right) z-B \mathcal{R}^{-1} B^{\top} \check{\varphi}+\left(F-B \mathcal{R}^{-1} D^{\top} P \widetilde{F}\right) \mathbb{E}[z]\right] d t } \\
&+\left[\left(C-D \mathcal{R}^{-1} \mathcal{P}\right) z-D \mathcal{R}^{-1} B^{\top} \check{\varphi}+\left(\widetilde{F}-D \mathcal{R}^{-1} D^{\top} P \widetilde{F}\right) \mathbb{E}[z]\right] d W_{1}(t), \\
& d \check{y}_{0}= {\left[H Q(I-\hat{H}) \mathbb{E}[z]-\widetilde{G}^{\top} \mathbb{E}\left[\check{\beta}_{1}^{1}\right]\right] d t, } \\
& d \check{y}_{1}= {\left[-Q z+Q \hat{H} \mathbb{E}[z]-A^{\top} \check{y}_{1}-C^{\top} \breve{\beta}_{1}^{1}\right] d t+\check{\beta}_{1}^{1} d W_{1}(t), } \\
& d \check{y}_{2}= {\left[\hat{H} Q(I-\hat{H}) \mathbb{E}[z]-F^{\top} \mathbb{E}\left[\check{y}_{1}\right]-\widetilde{F}^{\top} \mathbb{E}\left[\check{\beta}_{1}^{1}\right]-(A+F)^{\top} \check{y}_{2}\right] d t, } \\
& d \check{\varphi}=-\left\{\left[A^{\top}-\mathcal{P}^{\top} \mathcal{R}^{-1} B^{\top}\right] \check{\varphi}+\left[F-\left(\mathcal{P}^{\top} \mathcal{R}^{-1} D^{\top}-C^{\top}\right) P \widetilde{F}\right] \mathbb{E}[z]-S\right\} d t, \\
& S=Q \hat{H} \hat{x}+\hat{H} Q(\hat{x}-\hat{H} \hat{x})-F^{\top} y_{2}-F^{\top} \hat{y}_{1}-\widetilde{F}^{\top} \hat{\beta}_{1}, \\
& z_{0} \equiv 0, \quad \check{\phi} \equiv 0, \quad z_{0}(0)=\xi_{0}, \quad z(0)=\xi, \quad \check{y}_{0}(T)=0, \\
& \check{y}_{1}(T)=0, \quad \check{y}_{2}(T)=0, \quad \check{\phi}(T)=0, \quad \check{\varphi}(T)=0,
\end{aligned}\right.
$$

which is consistent with the result of [25, equation (33)]. Moreover, $\left(\Lambda_{1}, \Lambda_{2}\right)$ takes the following form

$$
\left\{\begin{array}{l}
\Lambda_{1}=-\left(R+D^{\top} P D\right)^{-1}\left(B^{\top} P+D^{\top} P C\right), \\
\Lambda_{2}=-\left(R+D^{\top} P D\right)^{-1}\left(B^{\top} \varphi+D^{\top} P \widetilde{F} \hat{x}\right),
\end{array}\right.
$$

which is also consistent with [25].

Through the discussion above, we compare our result with some previous literature. For the next part, we will study the performance of the mean-field strategy. Specifically, we will prove its asymptotic optimality. 


\section{ASYMPTOTIC $\varepsilon$-OPTIMALITY}

Definition 1: A mixed strategy set $\left\{u_{i}^{\varepsilon} \in \mathcal{U}_{i}\right\}_{i=0}^{N}$ is called asymptotically $\varepsilon$-optimal if there exists $\varepsilon=\varepsilon(N)>0$, $\lim _{N \rightarrow \infty} \varepsilon(N)=0$ such that

$$
\left\{\begin{array}{l}
\mathcal{J}_{0}\left(u_{0}^{\varepsilon}, u_{-0}^{\varepsilon}\right) \leq \inf _{u_{0} \in \mathcal{U}_{0}} \mathcal{J}_{0}\left(u_{0}, u_{-0}^{\varepsilon}\right)+\varepsilon, \\
\frac{1}{N}\left(\mathcal{J}_{\text {soc }}^{(N)}\left(u_{0}^{\varepsilon}, u_{-0}^{\varepsilon}\right)-\inf _{u_{-0} \in \mathcal{U}_{-0}} \mathcal{J}_{\text {soc }}^{(N)}\left(u_{0}^{\varepsilon}, u_{-0}\right)\right) \leq \varepsilon,
\end{array}\right.
$$

where $u_{-0}^{\varepsilon}:=\left\{u_{1}^{\varepsilon}, \cdots, u_{N}^{\varepsilon}\right\}$. In this case, $u_{0}^{\varepsilon}, u_{-0}^{\varepsilon}$ achieve an asymptotic $\varepsilon$-equilibrium, and $u_{1}^{\varepsilon}, \cdots, u_{N}^{\varepsilon}$ achieve an asymptotic $\varepsilon$-social optimum.

Let $\widetilde{u}$ be the mean-field strategy given in Section $\mathrm{V}$ and the realized decentralized states $\left(\widetilde{x}_{0}, \widetilde{x}_{1}, \cdots, \widetilde{x}_{N}\right)$ satisfy:

$$
\left\{\begin{aligned}
d \tilde{x}_{0}= & \left(A_{0} \tilde{x}_{0}+B_{0} \tilde{u}_{0}+F_{0} \tilde{x}^{(N)}\right) d t, \\
& +\left(C_{0} \tilde{x}_{0}+D_{0} \tilde{u}_{0}+\widetilde{F}_{0} \tilde{x}^{(N)}\right) d W_{0}, \quad \tilde{x}_{0}(0)=\xi_{0} \\
d \tilde{x}_{i}= & \left(A \tilde{x}_{i}+B \tilde{u}_{i}+F \tilde{x}^{(N)}\right) d t \\
& +\left[C \tilde{x}_{i}+D \tilde{u}_{i}+\widetilde{F} \tilde{x}^{(N)}+\widetilde{G} \tilde{x}_{0}\right] d W_{i}, \quad \tilde{x}_{i}(0)=\xi .
\end{aligned}\right.
$$

where $\widetilde{x}^{(N)}=\frac{1}{N} \sum_{i=1}^{N} \widetilde{x}_{i}$. First, we need some estimations. In the proofs below, we will use $K$ to denote a generic constant whose value may change from line to line.

Lemma 1: [11, Lemma 5.1] Under (H1)-(H3) and (SA), there exists a constant $K_{1}$ independent of $N$ such that

$$
\sup _{0 \leq i \leq N} \mathbb{E} \sup _{0 \leq t \leq T}\left\|\widetilde{x}_{i}(t)\right\|^{2}+\sup _{0 \leq i \leq N} \mathbb{E} \sup _{0 \leq t \leq T}\left\|\bar{z}_{i}(t)\right\|^{2} \leq K_{1}
$$

Lemma 2: Under (H1)-(H3) and (SA), there exists a constant $K_{2}$ independent of $N$ such that

$$
\mathbb{E} \sup _{0 \leq t \leq T}\left\|\widetilde{x}^{(N)}(t)-\mathbb{E}^{W_{0}}[z]\right\|^{2} \leq \frac{K_{2}}{N} .
$$

Proof: It is easy to get that

$$
\begin{aligned}
& d\left(\widetilde{x}^{(N)}-\mathbb{E}^{W_{0}}[z]\right) \\
= & (A+F)\left(\widetilde{x}^{(N)}-\mathbb{E}^{W_{0}}[z]\right)+B \Lambda_{1}\left(\frac{1}{N} \sum_{i=1}^{N} \bar{z}_{i}-\mathbb{E}^{W_{0}}[z]\right) d t \\
& +\frac{1}{N} \sum_{i=1}^{N}\left[C \widetilde{x}_{i}+D \tilde{u}_{i}+\widetilde{F} \widetilde{x}^{(N)}+\widetilde{G} \widetilde{x}_{0}\right] d W_{i} .
\end{aligned}
$$

Therefore,

$$
\begin{aligned}
& \mathbb{E} \sup _{0 \leq s \leq t}\left\|\widetilde{x}^{(N)}(s)-\mathbb{E}^{W_{0}}[z]\right\|^{2} \\
\leq & K \mathbb{E} \int_{0}^{t}\left\|\widetilde{x}^{(N)}-\mathbb{E}^{W_{0}}[z]\right\|^{2}+\left\|\frac{1}{N} \sum_{i=1}^{N} \bar{z}_{i}-\mathbb{E}^{W_{0}}[z]\right\|^{2} d s \\
& +\frac{K}{N^{2}} \mathbb{E} \sup _{0 \leq s \leq t}\left\|\int_{0}^{s} \sum_{i=1}^{N}\left[C \widetilde{x}_{i}+D \tilde{u}_{i}+\widetilde{F} \widetilde{x}^{(N)}+\widetilde{G} \widetilde{x}_{0}\right] d W_{i}\right\|^{2} .
\end{aligned}
$$

By

$$
\left\{\begin{array}{l}
d\left(\frac{1}{N} \sum_{i=1}^{N} \bar{z}_{i}-\mathbb{E}^{W_{0}}[z]\right)=\left(\left(A+B \Lambda_{1}\right)\left(\frac{1}{N} \sum_{i=1}^{N} \bar{z}_{i}-\mathbb{E}^{W_{0}}[z]\right)\right) d t \\
\quad+\frac{1}{N} \sum_{i=1}^{N}\left(\left(C+D \Lambda_{1}\right) \bar{z}_{i}+D \Lambda_{2}+\widetilde{F} \mathbb{E}^{W_{0}}[z]+\widetilde{G} \bar{z}_{0}\right) d W_{i} \\
\left(\frac{1}{N} \sum_{i=1}^{N} \bar{z}_{i}-\mathbb{E}^{W_{0}}[z]\right)(0)=0 .
\end{array}\right.
$$

it is easy to get

$$
\mathbb{E} \sup _{0 \leq s \leq t}\left\|\frac{1}{N} \sum_{i=1}^{N} \bar{z}_{i}-\mathbb{E}^{W_{0}}[z]\right\|^{2}=O\left(\frac{1}{N}\right) .
$$

Then by Burkholder-Davis-Gundy inequality, we have

$$
\begin{aligned}
& \mathbb{E} \sup _{0 \leq s \leq t}\left\|\widetilde{x}^{(N)}(s)-\mathbb{E}^{W_{0}}[z]\right\|^{2} \\
\leq & K \mathbb{E} \int_{0}^{t}\left\|\widetilde{x}^{(N)}-\mathbb{E}^{W_{0}}[z]\right\|^{2} d s \\
& +\frac{K}{N^{2}} \mathbb{E} \int_{0}^{t} \sum_{i=1}^{N}\left\|C \widetilde{x}_{i}+D \tilde{u}_{i}+\widetilde{F} \widetilde{x}^{(N)}+\widetilde{G} \widetilde{x}_{0}\right\|^{2} d s \\
\leq & K \mathbb{E} \int_{0}^{t}\left\|\widetilde{x}^{(N)}-\mathbb{E}^{W_{0}}[z]\right\|^{2} d s+\frac{K}{N}\left(1+\sup _{0 \leq i \leq N} \mathbb{E} \sup _{0 \leq t \leq T}\left\|\widetilde{x}_{i}(t)\right\|^{2}\right) .
\end{aligned}
$$

Finally, it follows from Gronwall's inequality, and Lemma 1 that there exists a constant $K_{2}$ independent of $N$ such that

$$
\mathbb{E} \sup _{0 \leq t \leq T}\left\|\widetilde{x}^{(N)}(t)-\mathbb{E}^{W_{0}}[z]\right\|^{2} \leq \frac{K_{2}}{N}
$$

Lemma 3: Under (H1)-(H3) and (SA), there exists a constant $K_{3}$ independent of $N$ such that

$$
\sup _{0 \leq i \leq N} \mathbb{E} \sup _{0 \leq t \leq T}\left\|\widetilde{x}_{i}(t)-\bar{z}_{i}(t)\right\|^{2} \leq \frac{K_{3}}{N} .
$$

Proof: It is easy to check that

$$
\begin{aligned}
d\left(\widetilde{x}_{i}-\bar{z}_{i}\right)= & {\left[A\left(\widetilde{x}_{i}-\bar{z}_{i}\right)+F\left(\widetilde{x}^{(N)}-\mathbb{E}^{W_{0}}[z]\right)\right] d t } \\
& +\left[C\left(\widetilde{x}_{i}-\bar{z}_{i}\right)+\widetilde{F}\left(\widetilde{x}^{(N)}-\mathbb{E}^{W_{0}}[z]\right)+\widetilde{G}\left(\widetilde{x}_{0}-\bar{z}_{0}\right)\right] d W_{i},
\end{aligned}
$$

and

$$
\begin{aligned}
& d\left(\widetilde{x}_{0}-\bar{z}_{0}\right) \\
= & {\left[A_{0}\left(\widetilde{x}_{0}-\bar{z}_{0}\right)+F_{0}\left(\widetilde{x}^{(N)}-\mathbb{E}^{W_{0}}[z]\right)\right] d t } \\
& +\left[C_{0}\left(\widetilde{x}_{0}-\bar{z}_{0}\right)+\widetilde{F}_{0}\left(\widetilde{x}^{(N)}-\mathbb{E}^{W_{0}}[z]\right)\right] d W_{0} .
\end{aligned}
$$

Therefore, it follows from Burkholder-Davis-Gundy inequality that

$$
\begin{aligned}
& \mathbb{E} \sup _{0 \leq s \leq t}\left\|\widetilde{x}_{i}(s)-\bar{z}_{i}(s)\right\|^{2} \\
& \leq K \mathbb{E} \int_{0}^{t}\left[\left\|\widetilde{x}_{i}-\bar{z}_{i}\right\|^{2}+\left\|\widetilde{x}^{(N)}-\mathbb{E}^{W_{0}}[z]\right\|^{2}\right] d s
\end{aligned}
$$

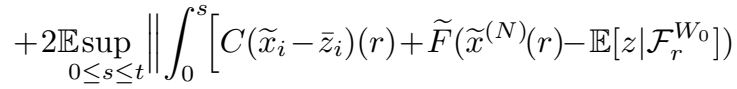

$$
\begin{aligned}
& \left.+\widetilde{G}\left(\widetilde{x}_{0}-\bar{z}_{0}\right)(r)\right] d W_{i}(r) \|^{2} \\
& \leq K \mathbb{E} \int_{0}^{t}\left\|\widetilde{x}_{i}-\bar{z}_{i}\right\|^{2} d s+K \mathbb{E} \int_{0}^{t}\left[\left\|\widetilde{x}^{(N)}-\mathbb{E}^{W_{0}}[z]\right\|^{2}+\left\|\widetilde{x}_{0}-\bar{z}_{0}\right\|^{2}\right] d s, \\
& \text { and } \\
& \mathbb{E} \sup _{0 \leq s \leq t}\left\|\widetilde{x}_{0}(s)-\bar{z}_{0}(s)\right\|^{2} \\
& \leq K \mathbb{E} \int_{0}^{t}\left[\left\|\widetilde{x}_{0}-\bar{z}_{0}\right\|^{2}+\left\|\widetilde{x}^{(N)}-\mathbb{E}^{W_{0}}[z]\right\|^{2}\right] d s \\
& +2 \mathbb{E} \sup _{0 \leq s \leq t} \| \int_{0}^{s}\left[C_{0}\left(\widetilde{x}_{0}-\bar{z}_{0}\right)(r)\right. \\
& \left.+\widetilde{F}_{0}\left(\widetilde{x}^{(N)}(r)-\mathbb{E}^{W_{0}}[z]\right)\right] d W_{0}(r) \|^{2} \\
& \leq K \mathbb{E} \int_{0}^{t}\left\|\widetilde{x}_{0}-\bar{z}_{0}\right\|^{2} d s+K \mathbb{E} \int_{0}^{t}\left\|\widetilde{x}^{(N)}-\mathbb{E}^{W_{0}}[z]\right\|^{2} d s .
\end{aligned}
$$


Therefore, it follows from Gronwall's inequality and Lemma 2 that

$$
\sup _{0 \leq i \leq N} \mathbb{E} \sup _{0 \leq t \leq T}\left\|\widetilde{x}_{i}(t)-\bar{z}_{i}(t)\right\|^{2} \leq \frac{K_{3}}{N}
$$

\section{A. Major agent}

Lemma 4: Under (H1)-(H3) and (SA),

$$
\left|\mathcal{J}_{0}\left(\widetilde{u}_{0}, \widetilde{u}_{-0}\right)-J_{0}\left(\bar{v}_{0}\right)\right|=O\left(\frac{1}{\sqrt{N}}\right) .
$$

Proof: Recall (3) and (8), it follows from

$$
\begin{aligned}
& \mathcal{J}_{0}\left(\widetilde{u}_{0}, \widetilde{u}_{-0}\right)-J_{0}\left(\bar{v}_{0}\right) \\
= & \frac{1}{2} \mathbb{E} \int_{0}^{T}\left\{\left\langle Q_{0}\left(\widetilde{x}_{0}-H_{0} \widetilde{x}^{(N)}\right), \widetilde{x}_{0}-H_{0} \widetilde{x}^{(N)}\right\rangle\right. \\
& \left.\quad-\left\langle Q_{0}\left(\bar{z}_{0}-H_{0} \mathbb{E}^{W_{0}}[z]\right), \bar{z}_{0}-H_{0} \mathbb{E}^{W_{0}}[z]\right\rangle\right\} d t \\
\leq & K \mathbb{E} \int_{0}^{T}\left[\left\|\widetilde{x}_{0}-\bar{z}_{0}\right\|^{2}+\left\|\widetilde{x}^{(N)}-\mathbb{E}^{W_{0}}[z]\right\|^{2}\right] d t \\
& +K \int_{0}^{T}\left[\left(\mathbb{E}\left\|\widetilde{x}_{0}-\bar{z}_{0}\right\|^{2}\right)^{\frac{1}{2}}+\left(\mathbb{E}\left\|\widetilde{x}^{(N)}-\mathbb{E}^{W_{0}}[z]\right\|^{2}\right)^{\frac{1}{2}}\right] d t=O\left(\frac{1}{\sqrt{N}}\right),
\end{aligned}
$$

where the last equality follows from Lemmas 1-3.

Consider the case when the major agent $\mathcal{A}_{0}$ uses an alternative strategy $u_{0}$ and the minor agent $\mathcal{A}_{i}$ still uses the strategy $\widetilde{u}_{i}$. The realized states with major agent's perturbation are

$$
\left\{\begin{array}{l}
d \alpha_{0}=\left(A_{0} \alpha_{0}+B_{0} u_{0}+F_{0} \alpha^{(N)}\right) d t+\left(C_{0} \alpha_{0}+D_{0} u_{0}+\widetilde{F}_{0} \alpha^{(N)}\right) d W_{0}, \\
d \alpha_{i}=\left[A \alpha_{i}+B \tilde{u}_{i}+F \alpha^{(N)}\right] d t+\left[C+D \tilde{u}_{i}+\widetilde{F} \alpha^{(N)}+\widetilde{G} \alpha_{0}\right] d W_{i}, \\
\alpha_{0}(0)=\xi_{0}, \quad \alpha_{i}(0)=\xi, \quad 1 \leq i \leq N,
\end{array}\right.
$$

where $\alpha^{(N)}=\frac{1}{N} \sum_{i=1}^{N} \alpha_{i}$. The decentralized limiting states with major agent's perturbation are

$$
\left\{\begin{aligned}
d \bar{\alpha}_{0}= & \left(A_{0} \bar{\alpha}_{0}+B_{0} u_{0}+F_{0} \mathbb{E}^{W_{0}}[z]\right) d t \\
& +\left(C_{0} \bar{\alpha}_{0}+D_{0} u_{0}+\widetilde{F}_{0} \mathbb{E}^{W_{0}}[z]\right) d W_{0}, \\
d \bar{\alpha}_{i}=\left[A \bar{\alpha}_{i}+B \tilde{u}_{i}+F \mathbb{E}^{W_{0}}[z]\right] d t & \\
& +\left[C \bar{\alpha}_{i}+D \tilde{u}_{i}+\bar{F} \mathbb{E}^{W_{0}}[z]+\widetilde{G} \bar{\alpha}_{0}\right] d W_{i}, \\
\bar{\alpha}_{0}(0)= & \xi_{0}, \quad \bar{\alpha}_{i}(0)=\xi, \quad 1 \leq i \leq N .
\end{aligned}\right.
$$

Lemma 5: Under (H1)-(H3) and (SA), we have

Proof:

$$
\left|\mathcal{J}_{0}\left(u_{0}, \widetilde{u}_{-0}\right)-J_{0}\left(u_{0}\right)\right|=O\left(\frac{1}{\sqrt{N}}\right) .
$$

$$
\begin{aligned}
& \mathcal{J}_{0}\left(u_{0}, \widetilde{u}_{-0}\right)-J_{0}\left(u_{0}\right) \\
= & \frac{1}{2} \mathbb{E} \int_{0}^{T}\left\{\left\langle Q_{0}\left(\alpha_{0}-H_{0} \alpha^{(N)}\right), \alpha_{0}-H_{0} \alpha^{(N)}\right\rangle\right. \\
& \left.\quad-\left\langle Q_{0}\left(\bar{\alpha}_{0}-H_{0} \mathbb{E}^{W_{0}}[z]\right), \bar{\alpha}_{0}-H_{0} \mathbb{E}^{W_{0}}[z]\right\rangle\right\} d t \\
\leq & K \mathbb{E} \int_{0}^{T}\left[\left\|\alpha_{0}-\bar{\alpha}_{0}\right\|^{2}+\left\|\alpha^{(N)}-\mathbb{E}^{W_{0}}[z]\right\|^{2}\right] d t \\
& +K \int_{0}^{T}\left[\left(\mathbb{E}\left\|\alpha_{0}-\bar{\alpha}_{0}\right\|^{2}\right)^{\frac{1}{2}}+\left(\mathbb{E}\left\|\alpha^{(N)}-\mathbb{E}^{W_{0}}[z]\right\|^{2}\right)^{\frac{1}{2}}\right] d t=O\left(\frac{1}{\sqrt{N}}\right) .
\end{aligned}
$$

The last equality is similar to Lemmas 1-3.

Theorem 2: Under (H1)-(H3) and (SA), $\tilde{u}_{0}$ is an asymptotically $\varepsilon$-optimal strategy for the major agent.

Proof: It follows from Lemma 4 and Lemma 5 that

$$
\begin{aligned}
\mathcal{J}_{0}\left(\widetilde{u}_{0}, \widetilde{u}_{-0}\right) & \leq J_{0}\left(\bar{v}_{0}\right)+O\left(\frac{1}{\sqrt{N}}\right) \leq J_{0}\left(u_{0}\right)+O\left(\frac{1}{\sqrt{N}}\right) \\
& \leq \mathcal{J}_{0}\left(u_{0}, \widetilde{u}_{-0}\right)+O\left(\frac{1}{\sqrt{N}}\right) .
\end{aligned}
$$

\section{B. Minor agents}

1) Representation of social cost: Rewrite the largepopulation system (1) and (2) as follows:

$$
d \mathbf{x}=(\mathbf{A} \mathbf{x}+\mathbf{B} u) d t+\sum_{i=0}^{N}\left(\mathbf{C}_{i} \mathbf{x}+\mathbf{D}_{i} u\right) d W_{i}, \quad \mathbf{x}(0)=\Xi,
$$

where

$$
\begin{aligned}
& \mathbf{A}=\left(\begin{array}{ccccc}
A_{0} & \frac{F_{0}}{N} & \frac{F_{0}}{N} & \cdots & \frac{F_{0}}{N} \\
0 & A+\frac{F}{N} & \frac{F}{N} & \cdots & \frac{F}{N} \\
0 & \frac{F}{N} & A+\frac{F}{N} & \cdots & \frac{F}{N} \\
\vdots & \vdots & \vdots & \ddots & \vdots \\
0 & \frac{F}{N} & \frac{F}{N} & \cdots & A+\frac{F}{N}
\end{array}\right), \mathbf{x}=\left(\begin{array}{c}
x_{0} \\
x_{1} \\
\vdots \\
x_{N}
\end{array}\right) \\
& \mathbf{B}=\left(\begin{array}{ccccc}
B_{0} & 0 & 0 & \cdots & 0 \\
0 & B & 0 & \cdots & 0 \\
0 & 0 & B & \cdots & 0 \\
\vdots & \vdots & \vdots & \ddots & \vdots \\
0 & 0 & 0 & \cdots & \dot{B}
\end{array}\right), u=\left(\begin{array}{c}
u_{0} \\
u_{1} \\
\vdots \\
u_{N}
\end{array}\right), \mathbf{C}_{0}=\left(\begin{array}{ccccc}
C_{0} & \widetilde{F}_{0} & \widetilde{F}_{0} & \cdots & \frac{\widetilde{F}_{0}}{N} \\
0 & 0 & 0 & \cdots & 0 \\
0 & 0 & 0 & \cdots & 0 \\
\vdots & \vdots & \vdots & \ddots & \vdots \\
0 & 0 & 0 & \cdots & 0
\end{array}\right) \\
& \mathbf{D}_{0}=\left(\begin{array}{ccccc}
D_{0} & 0 & 0 & \cdots & 0 \\
0 & 0 & 0 & \cdots & 0 \\
0 & 0 & 0 & \cdots & 0 \\
\vdots & \vdots & \vdots & \ddots & \vdots \\
0 & 0 & 0 & \cdots & 0
\end{array}\right), \mathbf{C}_{i}=\begin{array}{c}
1 \\
i+1 \\
\vdots \\
N+1
\end{array}\left(\begin{array}{ccccccc}
0 & \cdots & 0 & 0 & 0 & \cdots & 0 \\
\vdots & & \ddots & \vdots & \vdots & \vdots & \vdots \\
\frac{\tilde{\widetilde{F}}}{N} & \cdots & \frac{\widetilde{F}}{N} & \frac{\widetilde{F}}{N}+C & \frac{\tilde{\widetilde{F}}}{N} & \cdots & \frac{\tilde{\widetilde{F}}}{N} \\
\vdots & \vdots & \vdots & \vdots & & \ddots & \vdots \\
0 & \cdots & 0 & 0 & 0 & \cdots & 0
\end{array}\right), \\
& \mathbf{D}_{i}=\underset{i+1}{1} \begin{array}{c}
\vdots \\
N+1
\end{array}\left(\begin{array}{ccccccc}
0 & \cdots & 0 & 0 & 0 & \cdots & 0 \\
\vdots & & \ddots & \vdots & \vdots & \vdots & \vdots \\
0 & \cdots & 0 & \dot{D} & 0 & \cdots & 0 \\
\vdots & \vdots & \vdots & \vdots & \ddots & \vdots \\
0 & \cdots & 0 & 0 & 0 & \cdots & 0
\end{array}\right), \Xi=\left(\begin{array}{c}
\xi_{0} \\
\xi \\
\vdots \\
\xi
\end{array}\right) \text {. }
\end{aligned}
$$

Similarly, the social cost takes the following form:

$$
\begin{aligned}
& \mathcal{J}_{\text {soc }}^{(N)}(u) \\
& =\frac{1}{2} \sum_{i=1}^{n} \mathbb{E} \int_{0}^{T}\left[\left\langleQ\left(x_{i}-H x_{0}-\hat{H} x^{(N)}\right),\right.\right. \\
& \left.\left.\quad\left(x_{i}-H x_{0}-\hat{H} x^{(N)}\right)\right\rangle+\left\langle R u_{i}, u_{i}\right\rangle\right] d t \\
& =\frac{1}{2} \mathbb{E} \int_{0}^{T}[\langle\mathbf{Q} \mathbf{x}, \mathbf{x}\rangle+\langle\mathbf{R} u, u\rangle] d t,
\end{aligned}
$$

where

$$
\mathbf{Q}=\left(\begin{array}{ccccc}
\mathbf{Q}_{00} & \mathbf{Q}_{01} & \mathbf{Q}_{02} & \cdots & \mathbf{Q}_{0 N} \\
\mathbf{Q}_{10} & \mathbf{Q}_{11} & \mathbf{Q}_{12} & \cdots & \mathbf{Q}_{1 N} \\
\mathbf{Q}_{20} & \mathbf{Q}_{21} & \mathbf{Q}_{22} & \cdots & \mathbf{Q}_{2 N} \\
\vdots & \vdots & \vdots & \ddots & \vdots \\
\mathbf{Q}_{N 0} & \mathbf{Q}_{N 1} & \mathbf{Q}_{N 2} & \cdots & \mathbf{Q}_{N N}
\end{array}\right), \mathbf{R}=\left(\begin{array}{ccccc}
0 & 0 & 0 & \cdots & 0 \\
0 & R & 0 & \cdots & 0 \\
0 & 0 & R & \cdots & 0 \\
\vdots & \vdots & \vdots & \ddots & \vdots \\
0 & 0 & 0 & \cdots & R
\end{array}\right),
$$

and for $i=1, \cdots, N, j \neq i$,

$$
\left\{\begin{array}{l}
\mathbf{Q}_{00}=N Q+\hat{H}^{\top} Q \hat{H}-Q \hat{H}-\hat{H}^{\top} Q, \\
\mathbf{Q}_{0 i}=-\hat{H}^{\top} Q H+Q H, \\
\mathbf{Q}_{i 0}=-H Q \hat{H}+H Q, \\
\mathbf{Q}_{i i}=Q+\frac{1}{N}\left(\hat{H}^{\top} Q \hat{H}-Q \hat{H}-\hat{H}^{\top} Q\right), \mathbf{Q}_{i j}=\mathbf{Q}_{i i}-Q .
\end{array}\right.
$$

Next, define the following operators

$$
\left\{\begin{aligned}
&(L u(\cdot))(\cdot):=\Phi(\cdot)\left\{\int_{0} \Phi(s)^{-1}\left[\left(\mathbf{B}-\sum_{i=0}^{N} \mathbf{C}_{i} \mathbf{D}_{i}\right) u(s)\right] d s\right. \\
&\left.+\sum_{i=0}^{N} \int_{0}^{\cdot} \Phi(s)^{-1} \mathbf{D}_{i} u d W_{i}(s)\right\}, \\
& \widetilde{L} u(\cdot):=(L u(\cdot))(T), \quad \Gamma \Xi(\cdot):=\Phi(\cdot) \Phi^{-1}(0) \Xi, \quad \widetilde{\Gamma} \Xi:=(\Gamma \Xi)(T) .
\end{aligned}\right.
$$


Correspondingly, $L^{*}$ is defined as the adjoint operator of $L$. Hence, we can rewrite the cost functional as follows:

$$
\begin{aligned}
2 \mathcal{J}_{\text {soc }}^{(N)}(u)= & \left\langle\left(L^{*} \mathbf{Q} L+\mathbf{R}\right) u(\cdot), u(\cdot)\right\rangle+2\left\langle L^{*} Q \Gamma \Xi(\cdot), u(\cdot)\right\rangle \\
& +\langle Q \Gamma \Xi(\cdot), \Gamma \Xi(\cdot)\rangle \\
:= & \left\langle M_{2} u(\cdot), u(\cdot)\right\rangle+2\left\langle M_{1}, u(\cdot)\right\rangle+M_{0} .
\end{aligned}
$$

Note that, $M_{2}$ is a self-adjoint positive semidefinite bounded linear operator.

2) Minor agent's perturbation: Let us consider the case that the minor agent $\mathcal{A}_{i}$ uses an alternative strategy $u_{i}$ while the major agent and all other minor agents $\mathcal{A}_{j}, j \neq i$ use the strategies $\widetilde{u}_{-i}$. The realized states with the $i^{\text {th }}$ minor agent's perturbation are

$$
\left\{\begin{aligned}
d \check{x}_{0}= & {\left[A_{0} \check{x}_{0}+B_{0} \tilde{u}_{0}+F_{0} \check{x}^{(N)}\right] d t } \\
& +\left[C_{0} \check{x}_{0}+D_{0} \tilde{u}_{0}+\widetilde{F}_{0} \check{x}^{(N)}\right] d W_{0}, \\
d \check{x}_{i}= & {\left[A \check{x}_{i}+B u_{i}+F \check{x}^{(N)}\right] d t } \\
& +\left[C \check{x}_{i}+D u_{i}+\widetilde{F} \check{x}^{(N)}+\widetilde{G} \check{x}_{0}\right] d W_{i}, \\
d \check{x}_{j}= & {\left[A \check{x}_{j}+B \tilde{u}_{j}+F \check{x}^{(N)}\right] d t } \\
& +\left[C \check{x}_{j}+D \tilde{u}_{j}+\widetilde{F} \check{x}^{(N)}+\widetilde{G} \check{x}_{0}\right] d W_{j}, \\
y_{0}(0)= & \xi_{0}, \quad y_{i}(0)=\xi, \quad 1 \leq j \leq N, j \neq i,
\end{aligned}\right.
$$

where $\check{x}^{(N)}=\frac{1}{N} \sum_{i=1}^{N} \check{x}_{i}$. Moreover, introduce the following system:

$$
\left\{\begin{aligned}
\widetilde{l l}_{0}= & {\left[A_{0} \widetilde{l}_{0}+B_{0} \tilde{u}_{0}+F_{0} \mathbb{E}^{W_{0}}[z]\right] d t } \\
& +\left[C_{0} \widetilde{l}_{0}+D_{0} \tilde{u}_{0}+\widetilde{F}_{0} \mathbb{E}^{W_{0}}[z]\right] d W_{0}, \\
{\widetilde{d l_{j}}}_{j}= & {\left[A \widetilde{l}_{j}+B \tilde{u}_{j}+F \mathbb{E}^{W_{0}}[z]\right] d t } \\
& +\left[C \widetilde{l}_{j}+D \tilde{u}_{j}+\widetilde{F} \mathbb{E}^{W_{0}}[z]+\widetilde{G} z_{0}\right] d W_{j}, \\
\widetilde{l}_{0}(0)= & \xi_{0}, \quad \widetilde{l}_{j}(0)=\xi, \quad 1 \leq j \leq N,
\end{aligned}\right.
$$

and

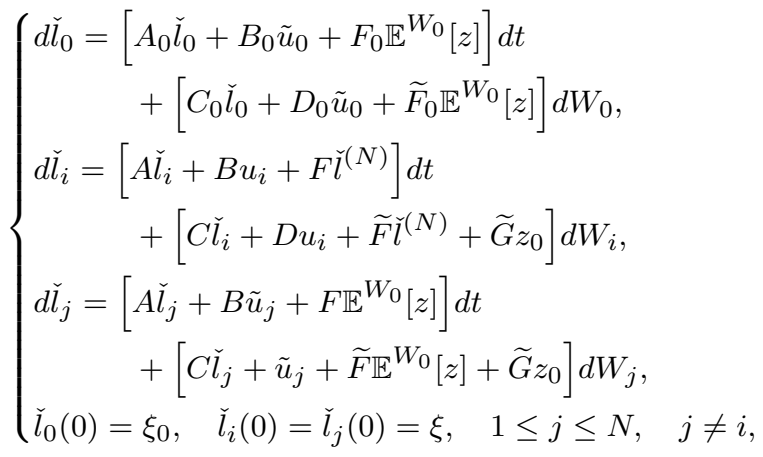

where $\widetilde{l}^{(N)}=\sum_{i=1}^{N} \frac{1}{N} \widetilde{l}_{i}$ and $\check{l}^{(N)}=\frac{1}{N} \sum_{i=1}^{N} \check{l}_{i}$. Similar to Lemma 1, we have

$$
\left\{\begin{array}{l}
\mathbb{E} \sup _{0 \leq t \leq T}\left\|\check{x}_{i}(t)\right\|^{2}+\mathbb{E} \sup _{0 \leq t \leq T}\left\|\check{l}_{i}(t)\right\|^{2} \leq K\left(1+\left\|u_{i}\right\|_{L^{2}}^{2}\right), \\
\mathbb{E} \sup _{0 \leq t \leq T}\left\|\widetilde{l}_{i}(t)\right\|^{2} \leq K .
\end{array}\right.
$$

Similar to the computation in Section IV-A we have

$$
\begin{aligned}
\delta \mathcal{J}_{\text {soc }}^{(N)}=\mathbb{E} \int_{0}^{T} & {\left[\left\langle Q \widetilde{x}_{i}, \delta x_{i}\right\rangle+\left\langle R \widetilde{u}_{i}, \delta u_{i}\right\rangle-\left\langle Q\left(\hat{H} \hat{x}+H z_{0}\right)\right.\right.} \\
+ & \hat{H} Q\left(\hat{x}-\hat{H} \hat{x}-H z_{0}\right)-F^{\top} y_{2}-F^{\top} \mathbb{E}^{W_{0}}\left[y_{1}^{j}\right] \\
& \left.\left.-\widetilde{F}^{\top} \mathbb{E}^{W_{0}}\left[\beta_{1}^{j j}\right]-F_{0}^{\top} y_{1}^{0}-\widetilde{F}_{0}^{\top} \beta_{1}^{0}, \delta x_{i}\right\rangle\right] d t+\sum_{l=1}^{13} \varepsilon_{l, i},
\end{aligned}
$$

where $\varepsilon_{1, i}, \cdots, \varepsilon_{13, i}$ are defined by (14), (16), (18) and (22).

Finally, we have

$\delta \mathcal{J}_{\text {soc }}^{(N)}=\mathbb{E} \int_{0}^{T}\left[\left\langle Q \widetilde{l}_{i}, \delta l_{i}\right\rangle-\left\langle S, \delta l_{i}\right\rangle+\left\langle R \widetilde{u}_{i}, \delta u_{i}\right\rangle\right] d t+\sum_{l=1}^{15} \varepsilon_{l, i}$,

where

$$
\left\{\begin{array}{l}
\varepsilon_{14}=\mathbb{E} \int_{0}^{T}\left[\left\langle Q\left(\widetilde{x}_{i}-\widetilde{l}_{i}\right), \delta x_{i}\right\rangle+\left\langle Q \widetilde{l}_{i}, \check{x}_{i}-\check{l}_{i}\right\rangle-\left\langle Q \widetilde{l}_{i}, \widetilde{x}_{i}-\widetilde{l}_{i}\right\rangle\right] d t, \\
\varepsilon_{15}=\mathbb{E} \int_{0}^{T}\left[\left\langle S, \check{x}_{i}-\check{l}_{i}\right\rangle-\left\langle S, \widetilde{x}_{i}-\widetilde{l}_{i}\right\rangle\right] d t .
\end{array}\right.
$$

At the end of this subsection, we give some lemmas which will be used in subsection 3 )

Lemma 6: Under (H1)-(H3) and (SA), there exists a constant $K_{9}$ independent of $N$ such that

$$
\mathbb{E} \sup _{0 \leq t \leq T}\left\|\check{x}^{(N)}(t)-\mathbb{E}^{W_{0}}[z]\right\|^{2} \leq K_{9}\left(\frac{1}{N}+\frac{\left\|u_{i}\right\|_{L^{2}}^{2}}{N^{2}}\right) .
$$

Proof: Note that

$$
\begin{aligned}
d \check{x}^{(N)}= & {\left[A \check{x}^{(N)}+\frac{1}{N} B \Lambda_{1} \sum_{j=1}^{N} \bar{z}_{j}-\frac{1}{N} B \Lambda_{1} \bar{z}_{i}+\frac{1}{N} B u_{i}+\frac{N-1}{N} B \Lambda_{2}\right.} \\
& \left.+F \check{x}^{(N)}\right] d t+\frac{1}{N}\left[C \check{x}_{i}+D u_{i}+\widetilde{F} \check{x}^{(N)}+\widetilde{G} \check{x}_{0}\right] d W_{i} \\
& +\frac{1}{N} \sum_{j \neq i}\left[C \check{x}_{j}+D \tilde{u}_{j}+\widetilde{F} \check{x}^{(N)}+\widetilde{G} \check{x}_{0}\right] d W_{j} \\
= & {\left[(A+F) \check{x}^{(N)}+\frac{1}{N} B \Lambda_{1} \sum_{j=1}^{N} \bar{z}_{j}+\frac{1}{N} B\left(u_{i}-\Lambda_{1} \bar{z}_{i}-\Lambda_{2}\right)\right.} \\
& \left.+B \Lambda_{2}\right] d t+\frac{1}{N}\left[C \check{x}_{i}+D u_{i}+\widetilde{F} \check{x}^{(N)}+\widetilde{G} \check{x}_{0}\right] d W_{i} \\
& +\frac{1}{N} \sum_{j \neq i}\left[C \check{x}_{j}+D \tilde{u}_{j}+\widetilde{F} \check{x}^{(N)}+\widetilde{G} \check{x}_{0}\right] d W_{j},
\end{aligned}
$$

and similar to [22, equation (5.7)] we have

$$
\begin{aligned}
d \mathbb{E}^{W_{0}}[z]= & {\left[\left(A-B \mathcal{R}^{-1}\left(B^{\top} P+D^{\top} P C\right)\right) \mathbb{E}^{W_{0}}[z]\right.} \\
& -B \mathcal{R}^{-1} D^{\top} P \widetilde{G} \mathbb{E}^{W_{0}}\left[z_{0}\right]-B \mathcal{R}^{-1} B^{\top} \mathbb{E}^{W_{0}}[\check{\varphi}] \\
& \left.-B \mathcal{R}^{-1} D^{\top} \mathbb{E}^{W_{0}}[\check{\eta}]+\left(F-B \mathcal{R}^{-1} D^{\top} P \widetilde{F}\right) \mathbb{E}^{W_{0}}[z]\right] d t
\end{aligned}
$$

Thus,

$$
\begin{aligned}
& d\left(\check{x}^{(N)}-\mathbb{E}^{W_{0}}[z]\right) \\
= & {\left[(A+F)\left(\check{x}^{(N)}-\mathbb{E}^{W_{0}}[z]\right)+B \Lambda_{1}\left(\frac{1}{N} \sum_{j=1}^{N} \bar{z}_{j}-\mathbb{E}^{W_{0}}(z)\right)\right.} \\
& \left.+\frac{1}{N} B\left(u_{i}-\Lambda_{1} \bar{z}_{i}-\Lambda_{2}\right)\right] d t \\
& +\frac{1}{N}\left[C \check{x}_{i}+D u_{i}+\widetilde{F} \check{x}^{(N)}+\widetilde{G} \check{x}_{0}\right] d W_{i} \\
& +\frac{1}{N} \sum_{j \neq i}\left[C \check{x}_{j}+D \tilde{u}_{j}+\widetilde{F} \check{x}^{(N)}+\widetilde{G} \check{x}_{0}\right] d W_{j} .
\end{aligned}
$$


By Burkholder-Davis-Gundy inequality and (34), we have

$$
\begin{aligned}
& \mathbb{E} \sup _{0 \leq s \leq t}\left\|\check{x}^{(N)}(s)-\mathbb{E}^{W_{0}}[z]\right\|^{2} \\
\leq & K \mathbb{E} \int_{0}^{t}\left[\left\|\check{x}^{(N)}-\mathbb{E}^{W_{0}}[z]\right\|^{2}+\frac{1}{N^{2}}\left\|\check{x}_{j}\right\|^{2}+\frac{1}{N^{2}}\left\|u_{i}\right\|^{2}+\frac{1}{N}\right] d s+O\left(\frac{1}{N}\right) \\
& +\frac{K}{N^{2}} \mathbb{E} \sup _{0 \leq s \leq t}\left\|\int_{0}^{s}\left[C \check{x}_{i}+D u_{i}+\widetilde{F} \check{x}^{(N)}+\widetilde{G} \check{x}_{0}\right] d W_{i}\right\|^{2} \\
& +\frac{K}{N^{2}} \mathbb{E} \sup _{0 \leq s \leq t}\left\|\sum_{j \neq i} \int_{0}^{s}\left[C \check{x}_{j}+D \tilde{u}_{j}+\widetilde{F}_{\check{x}^{(N)}}+\widetilde{G}_{\check{x}_{0}}\right] d W_{j}\right\|^{2} \\
\leq & K \mathbb{E} \int_{0}^{t}\left\|\check{x}^{(N)}-\mathbb{E}^{W_{0}}[z]\right\|^{2} d s+O\left(\frac{1}{N}\right)+\frac{\left\|u_{i}\right\|_{L^{2}}^{2}}{N^{2}} .
\end{aligned}
$$

Finally, it follows from Gronwall's inequality that

$$
\mathbb{E} \sup _{0 \leq t \leq T}\left\|\check{x}^{(N)}(t)-\mathbb{E}^{W_{0}}[z]\right\|^{2} \leq K_{9}\left(\frac{1}{N}+\frac{\left\|u_{i}\right\|_{L^{2}}^{2}}{N^{2}}\right) .
$$

By Lemma 2 and Lemma 6, we have

Lemma 7: Under (H1)-(H3) and (SA), there exist constants $K_{10}, K_{11}, K_{12}$ independent of $N$ such that

$$
\begin{aligned}
& \mathbb{E} \sup _{0 \leq t \leq T}\left\|\check{x}_{0}(t)-z_{0}(t)\right\|^{2} \leq K_{10}\left(\frac{1}{N}+\frac{\left\|u_{i}\right\|_{L^{2}}^{2}}{N^{2}}\right), \\
& \sup _{0 \leq j \leq N} \mathbb{E} \sup _{0 \leq t \leq T}\left\|\check{x}_{j}(t)-\check{l}_{j}(t)\right\|^{2} \leq K_{11}\left(\frac{1}{N}+\frac{\left\|u_{i}\right\|_{L^{2}}^{2}}{N^{2}}\right), \\
& \sup _{0 \leq j \leq N} \mathbb{E} \sup _{0 \leq t \leq T}\left\|\widetilde{x}_{j}(t)-\widetilde{l}_{j}(t)\right\|^{2} \leq K_{12}\left(\frac{1}{N}+\frac{\left\|u_{i}\right\|_{L^{2}}^{2}}{N^{2}}\right) . \\
& \text { Lemma } 8: \text { Under (H1)-(H3) and (SA), there exists a con- }
\end{aligned}
$$
stant $K_{13}$ independent of $N$ such that

$$
\sup _{0 \leq j \leq N, j \neq i} \mathbb{E} \sup _{0 \leq t \leq T}\left\|\delta x_{j}\right\|^{2} \leq K_{13}\left(\frac{1}{N^{2}}+\frac{\left\|u_{i}\right\|_{L^{2}}^{2}}{N^{2}}\right) .
$$

Proof: By the equations of $\delta x_{0}, \delta x_{i}, \delta x_{j}$ and Gronwall's inequality, we have

$$
\begin{aligned}
& \mathbb{E} \sup _{0 \leq s \leq t}\left\|\delta x_{0}(s)\right\|^{2} \leq K \mathbb{E} \int_{0}^{t}\left\|\delta x^{(N)}\right\|^{2} d s, \\
& \sup _{1 \leq j \leq N, j \neq i} \mathbb{E} \sup _{0 \leq s \leq t}\left\|\delta x_{j}(s)\right\|^{2} \leq K \mathbb{E} \int_{0}^{t}\left\|\delta x^{(N)}\right\|^{2} d s, \\
& \mathbb{E} \sup _{0 \leq s \leq t}\left\|\delta x_{i}(s)\right\|^{2} \leq K+K \mathbb{E} \int_{0}^{t}\left\|\delta x^{(N)}\right\|^{2} d s .
\end{aligned}
$$

It is easy to check that

$$
\begin{aligned}
d\left(\frac{1}{N} \sum_{j=1}^{N} \delta x_{j}\right)=[ & \left.A\left(\frac{1}{N} \sum_{j=1}^{N} \delta x_{j}\right)+\frac{1}{N} B \delta u_{i}+F \delta x^{(N)}\right] d t \\
& +\frac{1}{N} \sum_{j=1}^{N}\left[C \delta x_{j}+\widetilde{F} \delta x^{(N)}+\widetilde{G} \delta x_{0}\right] d W_{j} .
\end{aligned}
$$

Applying Itô's formula to $\left\|\sum_{j=1}^{N} \delta x_{j}\right\|^{2}$, we have

$$
\begin{aligned}
\mathbb{E}\left\|\frac{1}{N} \sum_{j=1}^{N} \delta x_{j}\right\|^{2} \leq & K \mathbb{E} \int_{0}^{t}\left\|\frac{1}{N} \sum_{j=1}^{N} \delta x_{j}\right\|^{2}+\frac{1}{N^{2}}\left\|\delta u_{i}\right\|_{L^{2}}^{2} d s \\
& +\frac{1}{N^{2}} \sum_{j=1}^{N} \mathbb{E} \int_{0}^{t}\left[C \delta x_{j}+\widetilde{F} \delta x^{(N)}+\widetilde{G} \delta x_{0}\right]^{2} d s \\
\leq & K \mathbb{E} \int_{0}^{t}\left\|\frac{1}{N} \sum_{j=1}^{N} \delta x_{j}\right\|^{2}+K\left(\frac{1}{N^{2}}+\frac{\left\|\delta u_{i}\right\|_{L^{2}}^{2}}{N^{2}}\right) .
\end{aligned}
$$

Moreover, we have

$$
\mathbb{E} \sup _{0 \leq t \leq T}\left\|\delta x_{0}(t)\right\|^{2} \leq K\left(\frac{1}{N^{2}}+\frac{\left\|\delta u_{i}\right\|_{L^{2}}^{2}}{N^{2}}\right)
$$

and

$$
\sup _{1 \leq j \leq N, j \neq i} \mathbb{E} \sup _{0 \leq t \leq T}\left\|\delta x_{j}(t)\right\|^{2} \leq K\left(\frac{1}{N^{2}}+\frac{\left\|\delta u_{i}\right\|_{L^{2}}^{2}}{N^{2}}\right) .
$$

Lemma 9: There exist constants $K_{14}, K_{15}, K_{16}$ independent of $N$ such that

$$
\begin{aligned}
& \mathbb{E} \sup _{0 \leq t \leq T}\left\|x^{* *}-\delta x_{-(0, i)}\right\|^{2} \leq K_{14}\left(\frac{1}{N}+\frac{\left\|\delta u_{i}\right\|_{L^{2}}^{2}}{N}\right), \\
& \mathbb{E} \sup _{0 \leq t \leq T}\left\|x_{0}^{*}-N \delta x_{0}\right\|^{2} \leq K_{15}\left(\frac{1}{N}+\frac{\left\|\delta u_{i}\right\|_{L^{2}}^{2}}{N}\right), \\
& \mathbb{E} \sup _{0 \leq t \leq T}\left\|x_{j}^{*}-N \delta x_{j}\right\|^{2} \leq K_{16}\left(\frac{1}{N}+\frac{\left\|\delta u_{i}\right\|_{L^{2}}^{2}}{N}\right) .
\end{aligned}
$$

Proof: First, we have the following dynamics

$$
\left\{\begin{aligned}
& d\left(x^{* *}-\delta x_{-(0, i)}\right) \\
= & {\left[(A+F)\left(x^{* *}-\delta x_{-(0, i)}\right)+\frac{1}{N}\left(F \delta x_{i}+\delta x_{-(0, i)}\right)\right] d t } \\
& -\sum_{j \neq i}\left[C \delta x_{j}+\widetilde{F} \delta x^{(N)}+\widetilde{G} \delta x_{0}\right] d W_{j}, \\
& d\left(x_{0}^{*}-N \delta x_{0}\right) \\
= & {\left[A_{0}\left(x_{0}^{*}-N \delta x_{0}\right)+F_{0}\left(x^{* *}-\delta x_{-(0, i)}\right)\right] d t } \\
& +\left[C_{0}\left(x_{0}^{*}-N \delta x_{0}\right)+\widetilde{F}_{0}\left(x^{* *}-\delta x_{-(0, i)}\right)\right] d W_{0}, \\
& d\left(x_{j}^{*}-N \delta x_{j}\right) \\
= & {\left[A\left(x_{j}^{*}-N \delta x_{j}\right)+F\left(x^{* *}-\delta x_{-(0, i)}\right)\right] d t } \\
& +\left[C\left(x_{j}^{*}-N \delta x_{j}\right)+\widetilde{F}\left(x^{* *}-\delta x_{-(0, i)}\right)\right. \\
& \left.\quad+\widetilde{G}\left(x_{0}^{*}-N \delta x_{0}\right)\right] d W_{j} .
\end{aligned}\right.
$$

Therefore, it follows from Burkholder-Davis-Gundy inequality that

$$
\begin{aligned}
& \mathbb{E} \sup _{0 \leq s \leq t}\left\|x^{* *}(s)-\delta x_{-(0, i)}(s)\right\|^{2} \\
\leq & K \mathbb{E} \int_{0}^{t}\left[\left\|x^{* *}(s)-\delta x_{-(0, i)}(s)\right\|^{2}+\frac{1}{N^{2}}\left\|F \delta x_{i}+\delta x_{-(0, i)}\right\|^{2}\right] d s \\
& +K \mathbb{E} \sup _{0 \leq s \leq t}\left\|\sum_{j \neq i} \int_{0}^{s}\left(\delta x_{j}+\delta x^{(N)}+\delta x_{0}\right) d W_{j}\right\|^{2} \\
\leq & K \mathbb{E} \int_{0}^{t}\left\|x^{* *}(s)-\delta x_{-(0, i)}(s)\right\|^{2} d s \\
+ & K \mathbb{E} \int_{0}^{t}\left[\frac{1}{N^{2}}\left\|F \delta x_{i}+\delta x_{-(0, i)}\right\|^{2}+\sum_{j \neq i}\left\|\delta x_{j}\right\|^{2}+\left\|\delta x^{(N)}\right\|^{2}+\left\|\delta x_{0}\right\|^{2}\right] d s .
\end{aligned}
$$

It then follows from Gronwall's inequality and Lemma 8 that

$$
\mathbb{E} \sup _{0 \leq t \leq T}\left\|x^{* *}(t)-\delta x_{-(0, i)}(t)\right\|^{2} \leq K\left(\frac{1}{N}+\frac{\left\|\delta u_{i}\right\|_{L^{2}}^{2}}{N}\right) .
$$

Similarly, we have the other two estimations. 
3) Asymptotic optimality: In order to prove asymptotic optimality for the minor agents, it suffices to consider the perturbations $u_{-0} \in \mathcal{U}_{-0}$ such that $\mathcal{J}_{\text {soc }}^{(N)}\left(\widetilde{u}_{0}, u_{-0}\right) \leq \mathcal{J}_{\text {soc }}^{(N)}\left(\widetilde{u}_{0}, \widetilde{u}_{-0}\right)$. It is easy to check that $\mathcal{J}_{\text {soc }}^{(N)}\left(\widetilde{u}_{0}, \widetilde{u}_{-0}\right) \leq K N$, where $K$ is a constant independent of $N$. Therefore, in what follows, we only consider the perturbations $u_{-0} \in \mathcal{U}_{-0}$ satisfying $\sum_{j=1}^{N} \mathbb{E} \int_{0}^{T}\left\|u_{j}\right\|^{2} d t \leq K N$. Let $\delta u_{i}=u_{i}-\widetilde{u}_{i}$, and consider a perturbation $u=\widetilde{u}+\left(0, \delta u_{1}, \cdots, \delta u_{N}\right):=\widetilde{u}+\delta u$. Then by Section VII-B.1, we have

$$
\begin{gathered}
2 \mathcal{J}_{\text {soc }}^{(N)}(\widetilde{u}+\delta u)=\left\langle M_{2}(\widetilde{u}+\delta u), \widetilde{u}+\delta u\right\rangle+2\left\langle M_{1}, \widetilde{u}+\delta u\right\rangle+M_{0} \\
=2 \mathcal{J}_{\text {soc }}^{(N)}(\widetilde{u})+2\left\langle M_{2} \widetilde{u}+M_{1}, \delta u\right\rangle+\left\langle M_{2} \delta u, \delta u\right\rangle,
\end{gathered}
$$

where $\left\langle M_{2} \widetilde{u}+M_{1}, \delta u_{i}\right\rangle$ is the Fréchet differential of $\mathcal{J}_{\text {soc }}^{(N)}$ on $\widetilde{u}$ with variation $\delta u_{i}$. Therefore, in order to prove asymptotic optimality for the minor agents, we only need to show that $\left\langle M_{2} \widetilde{u}+M_{1}, \delta u\right\rangle=o(N)$. To this end, we introduce another assumption:

(H4) There exists constants $L_{1}, L_{2}>0$ independent of $N$ such that

$$
\begin{aligned}
& \mathbb{E} \int_{0}^{T}\left\|\mathbb{E}^{W_{0}}\left[y_{1}^{1}\right]-\frac{1}{N} \sum_{j \neq i} y_{1}^{j}\right\|^{2} d t \leq \frac{L_{1}}{N}, \\
& \mathbb{E} \int_{0}^{T}\left\|\mathbb{E}^{W_{0}}\left[\beta_{1}^{11}\right]-\frac{1}{N} \sum_{j \neq i} \beta_{1}^{j j}\right\|^{2} d t \leq \frac{L_{2}}{N} .
\end{aligned}
$$

Theorem 3: Under (H1)-(H4) and (SA), $\left(\tilde{u}_{1}, \cdots, \tilde{u}_{N}\right)$ is an asymptotically $\varepsilon$-optimal strategy set for the minor agents whose individual cost functionals are given by (4) and the social cost is given by (5).

Proof: From Section VII-B.2, we have

$$
\begin{aligned}
& \left\langle M_{2} \widetilde{u}+M_{1}, \delta u\right\rangle \\
= & \sum_{i=1}^{N} \mathbb{E} \int_{0}^{T}\left[\left\langle Q \widetilde{l}_{i}, \delta l_{i}\right\rangle-\left\langle S, \delta l_{i}\right\rangle+\left\langle R \widetilde{u}_{i}, \delta u_{i}\right\rangle\right] d t+\sum_{i=1}^{N} \sum_{l=1}^{15} \varepsilon_{l},
\end{aligned}
$$

From the optimality of $\widetilde{u}$, we have

$$
\mathbb{E} \int_{0}^{T}\left[\left\langle Q \widetilde{l}_{i}, \delta l_{i}\right\rangle-\left\langle S, \delta l_{i}\right\rangle+\left\langle R \widetilde{u}_{i}, \delta u_{i}\right\rangle\right] d t=0 .
$$

Moreover, it follows from Lemma 19 and (H4) that

$$
\sum_{i=1}^{N} \sum_{l=1}^{15} \varepsilon_{l, i}=O(\sqrt{N}) \text {. }
$$

Therefore, $\left\langle M_{2} \widetilde{u}+M_{1}, \delta u\right\rangle=O(\sqrt{N})$.

$$
\begin{aligned}
& \text { Remark 7: Note that } \\
& \qquad \begin{array}{l}
d\left(\frac{1}{N} \sum_{j \neq i} \widetilde{x}_{j}-\mathbb{E}^{W_{0}}\left[\widetilde{x}_{1}\right]\right) \\
=\left[A\left(\frac{1}{N} \sum_{j \neq i} \widetilde{x}_{j}-\mathbb{E}^{W_{0}}\left[\widetilde{x}_{1}\right]\right)+F\left(\widetilde{x}^{(N)}-\mathbb{E}^{W_{0}}\left[\widetilde{x}^{(N)}\right]\right)\right. \\
\left.+B \Lambda_{1}\left(\frac{1}{N} \sum_{j \neq i} \bar{z}_{j}-\mathbb{E}^{W_{0}}\left[\bar{z}_{1}\right]\right)-\frac{1}{N} B \Lambda_{2}-\frac{1}{N} F \tilde{x}^{(N)}\right] d t \\
+\frac{1}{N} \sum_{j \neq i}\left[C \widetilde{x}_{j}+D \tilde{u}_{j}+\widetilde{F} \tilde{x}^{(N)}+\widetilde{G} \widetilde{x}_{0}\right] d W_{j} .
\end{array}
\end{aligned}
$$

Therefore, it follows from Burkholder-Davis-Gundy inequality and Gronwall's inequality that

$$
\mathbb{E} \sup _{0 \leq s \leq t}\left\|\frac{1}{N} \sum_{j \neq i} \widetilde{x}_{j}(s)-\mathbb{E}^{W_{0}}\left[\widetilde{x}_{1}\right]\right\|^{2} \leq \frac{K}{N} .
$$

If $C=0$, applying Itô's formula to $\left\|\frac{1}{N} \sum_{j \neq i} y_{1}^{j}-\mathbb{E}^{W_{0}}\left[y_{1}^{1}\right]\right\|^{2}$, it is easy to check that (39) in (H4) holds.

Remark 8: If the state has the following form

$$
\left\{\begin{array}{l}
d x_{0}=\left(A_{0} x_{0}+B_{0} u_{0}+F_{0} x^{(N)}\right) d t+\left(C_{0} x_{0}+D_{0} u_{0}+\widetilde{F}_{0} x^{(N)}\right) d W_{0}, \\
d x_{i}=\left(A x_{i}+B u_{i}+F x^{(N)}+G x_{0}\right) d t+D d W_{i}, \\
x_{0}(0)=\xi_{0}, x_{i}(0)=\xi, 1 \leq i \leq N .
\end{array}\right.
$$

Assumption (H4) is not needed to obtain the asymptotic optimality of the minor agents. However, if the state equations of the minor agents take the form (2), we need to suppose the assumption (H4) hold and we will continue to study this in the future work.

Last, by combining Theorems 2, 3 and recalling the Definition 1 , we have the following result

Theorem 4: The mean-field strategies $\tilde{u}_{0}, \tilde{u}_{-0}$ achieve an asymptotic $\varepsilon$-equilibrium between the major agent and the aggregation of minor agents, where $\tilde{u}_{0}=\Theta_{1} \tilde{x}_{0}+\Theta_{2}, \tilde{u}_{-0}=$ $\left(\tilde{u}_{1}, \cdots, \tilde{u}_{N}\right)$ and $\tilde{u}_{i}=\Lambda_{1} \tilde{x}_{i}+\Lambda_{2}$. Moreover $\tilde{u}_{1}, \cdots, \tilde{u}_{N}$ achieve an asymptotic $\varepsilon$-social optimum among the aggregation of minor agents. Thus, $\left(\tilde{u}_{0}, \tilde{u}_{-0}\right)$ is asymptotically $\varepsilon$ optimal.

Proof: By Theorem 2, we have

$$
\mathcal{J}_{0}\left(\tilde{u}_{0}, \tilde{u}_{-0}\right) \leq \inf _{u_{0} \in \mathcal{U}_{0}} \mathcal{J}_{0}\left(u_{0}, \tilde{u}_{-0}\right)+O\left(\frac{1}{\sqrt{N}}\right) .
$$

Moreover, by Theorem 3, we have

$$
\frac{1}{N}\left(\mathcal{J}_{\text {soc }}^{(N)}\left(\tilde{u}_{0}, \tilde{u}_{-0}\right)-\inf _{u_{-0} \in \mathcal{U}_{-0}} \mathcal{J}_{\text {soc }}^{(N)}\left(\tilde{u}_{0}, u_{-0}\right)\right) \leq O\left(\frac{1}{\sqrt{N}}\right) .
$$

Thus, by Definition 1, Theorem 4 holds straightforwardly.

\section{NUMERICAL ANALYSIS}

This section presents some numerical example to illustrate our theoretical results. Our example is motivated by an electric charging control problem in presence of distributed information network. Relevant literature include e.g., [7] and [19].

Consider two competitive charging providers in a powergrid network for given city. One provider (namely, the major agent in our model) still retains the traditional charging scheme upon centralized information, whereby its charging strategy is determined by a central controller. On the other hand, another provider, taking account the well-recognized distributed datum, prefers to adopt some decentralized charging scheme, where the strategy is determined by each distributed charging unit on grid-node, only upon their decentralized information on or around that node. In this case, such distributed provider is actually formalized into a cooperative team wherein all its sub-units or nodes acts as the minor agents as in our model.

Moreover, under some mild conditions on (linear) demandsupply curve, above competitive problem may be fit into a linear-quadratic setup whenever a quadratic deviation or tracing criteria is applied, as in [6] and [8]. Thus, we cook one example in our theoretical framework.

We now specify such example in details, by randomly generate its coefficients: $A_{0}=\left(\begin{array}{lll}0.6423 & 0.2057 \\ 0.0287 & 0.7907\end{array}\right), F_{0}=\left(\begin{array}{ll}0.9225 & 0.3780 \\ 0.6933 & 0.8048\end{array}\right)$, $C_{0}=\left(\begin{array}{ll}0.0125 & 0.4517 \\ 0.4720 & 0.1117\end{array}\right), \widetilde{F}_{0}=\left(\begin{array}{lll}0.8084 & 0.4284 \\ 0.7032 & 0.1955\end{array}\right), A=\left(\begin{array}{ll}0.1023 & 0.2995 \\ 0.1027 & 0.9415\end{array}\right), F=$ $\left(\begin{array}{ll}0.0377 & 0.8910 \\ 0.2866 & 0.1003\end{array}\right), C=\left(\begin{array}{ll}0.1641 & 0.0360 \\ 0.3271 & 0.8063\end{array}\right), \widetilde{F}=\left(\begin{array}{ll}0.3751 & 0.6241 \\ 0.4491 & 0.5093\end{array}\right), \widetilde{G}(\cdot)=$ $\left(\begin{array}{ll}0.5018 & 0.7881 \\ 0.6989 & 0.1633\end{array}\right), B_{0}=\left(\begin{array}{lll}0.45140 & 0.2916 \\ 0.4309 & 0.9989\end{array}\right), D_{0}(\cdot)=\left(\begin{array}{lll}0.4514 & 0.2916 \\ 0.4309 & 0.9989\end{array}\right)$, $B=\left(\begin{array}{ll}0.4389 & 0.4766 \\ 0.2411 & 0.3539\end{array}\right), D=\left(\begin{array}{ll}0.8756 & 0.9451 \\ 0.7493 & 0.8354\end{array}\right), Q_{0}=\left(\begin{array}{cc}0.6210 & 0 \\ 0 & 0.8691\end{array}\right)$, $H_{0}=\left(\begin{array}{cc}0.3250 & 0 \\ 0 & 0.5957\end{array}\right), Q=\left(\begin{array}{ccc}0.8701 & 0 \\ 0 & 0.1925\end{array}\right), H=\left(\begin{array}{ccc}0.3865 & 0 \\ 0 & 0.2957\end{array}\right)$, $\hat{H}=\left(\begin{array}{cc}0.7027 & 0 \\ 0 & 0.0354\end{array}\right), R_{0}=\left(\begin{array}{ccc}0.7160 & 0 \\ 0 & 0.5594\end{array}\right), R=\left(\begin{array}{ccc}0.3885 & 0 \\ 0 & 0.4182\end{array}\right)$, 


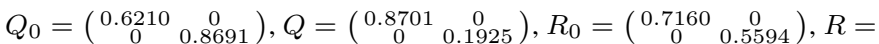
$\left(\begin{array}{cc}0.3885 & 0 \\ 0 & 0.4182\end{array}\right)$. It is easy to see that such generated coefficients are constants and surely $L^{\infty}$ and Lipschitz continuous. Thus, assumption (H1)-(H2) hold.

In the following simulation, we will calculate the feedback form mean-field strategies and also the corresponding state trajectories of major and minor agents. The convergence of the population average will be also simulated.

Firstly, we solve (28) by decentralizing method and decoupling method. As we mentioned above, the CC system can be rewritten as (30). Then by letting $\mathbb{X}_{1}:=\mathbb{E}\left[X \mid \mathcal{F}_{t}^{W_{0}}\right]$, $\mathbb{Y}_{1}:=\mathbb{E}\left[Y \mid \mathcal{F}_{t}^{W_{0}}\right], \mathbb{X}_{2}:=\left(X-\mathbb{X}_{1}\right), \mathbb{Y}_{2}:=\left(Y-\mathbb{Y}_{1}\right)$.

$$
\begin{aligned}
& \left\{\begin{array}{l}
d \mathbb{X}_{1}=\left[\left(\mathbb{A}_{1}+\overline{\mathbb{A}}_{1}\right) \mathbb{X}_{1}+\mathbb{B}_{1} \mathbb{Y}_{1}+\mathbb{F}_{1} \mathbb{E}\left[Z_{1} \mid \mathcal{F}_{t}^{W_{0}}\right]\right] d t \\
+\left[\left(\mathbb{C}_{1}^{0}+\overline{\mathbb{C}}_{1}^{0}\right) \mathbb{X}_{1}+\mathbb{D}_{1}^{0} E \mathbb{Y}_{1}+\mathbb{F}_{1}^{0} \mathbb{E}\left[Z_{1} \mid \mathcal{F}_{t}^{W_{0}}\right]\right] d W_{0},
\end{array}\right. \\
& d \mathbb{X}_{2}=\left[\mathbb{A}_{1} \mathbb{X}_{2}+\mathbb{B}_{1} \mathbb{Y}_{2}+\mathbb{F}_{1}\left(Z_{1}-\mathbb{E}\left[Z_{1} \mid \mathcal{F}_{t}^{W_{0}}\right]\right)\right] d t \\
& +\left[\mathbb{C}_{1}^{0} \mathbb{X}_{2}+\mathbb{D}_{1}^{0} \mathbb{Y}_{2}+\mathbb{F}_{1}^{0}\left(Z_{1}-\mathbb{E}\left[Z_{1} \mid \mathcal{F}_{t}^{W_{0}}\right]\right)\right] d W_{0} \\
& +\left[\mathbb{C}_{1}^{1} \mathbb{X}_{2}+\left(C_{1}^{1}+\overline{\mathbb{C}}_{1}^{1}\right) \mathbb{X}_{1}+\mathbb{D}_{1}^{1} \mathbb{Y}_{2}+\mathbb{D}_{1}^{1} \mathbb{Y}_{1}\right. \\
& \left.+\mathbb{F}_{1}^{1}\left(Z_{1}-\mathbb{E}\left[Z_{1} \mid \mathcal{F}_{t}^{W_{0}}\right]\right)+\mathbb{F}_{1}^{1} \mathbb{E}\left[Z_{1} \mid \mathcal{F}_{t}^{W_{0}}\right]\right] d W_{1} \\
& d \mathbb{Y}_{1}=\left[\left(\mathbb{A}_{2}+\overline{\mathbb{A}}_{2}\right) \mathbb{X}_{1}+\left(\mathbb{B}_{2}+\overline{\mathbb{B}}_{2}\right) \mathbb{Y}_{1}+\mathbb{C}_{2} \mathbb{E}\left[Z_{1} \mid \mathcal{F}_{t}^{W_{0}}\right]\right. \\
& \left.+\left(\tilde{\mathbb{C}}_{2}+\overline{\mathbb{C}}_{2}\right) \mathbb{E}\left[Z_{2} \mid \mathcal{F}_{t}^{W_{0}}\right]\right] d t+\mathbb{E}\left[Z_{1} \mid \mathcal{F}_{t}^{W_{0}}\right] d W_{0}, \\
& d \mathbb{Y}_{2}=\left[\mathbb{A}_{2} \mathbb{X}_{2}+\mathbb{B}_{2} \mathbb{Y}_{2}+\mathbb{C}_{2}\left(Z_{1}-\mathbb{E}\left[Z_{1} \mid \mathcal{F}_{t}^{W_{0}}\right]\right)\right. \\
& \left.+\tilde{\mathbb{C}}_{2}\left(Z_{2}-\mathbb{E}\left[Z_{2} \mid \mathcal{F}_{t}^{W_{0}}\right]\right)\right] d t+\left(Z_{1}-\mathbb{E}\left[Z_{1} \mid \mathcal{F}_{t}^{W_{0}}\right]\right) d W_{0}+Z_{2} d W_{1}, \\
& X(0)=\left(\xi_{0}^{\top}, \xi^{\top}\right)^{\top}, \quad Y(T)=\left(0^{\top}, 0^{\top}, 0^{\top}, 0^{\top}, 0^{\top}\right)^{\top} .
\end{aligned}
$$

Further, by letting $\mathbb{E}\left[Z_{1} \mid \mathcal{F}_{t}^{W_{0}}\right]=\mathbb{Z}_{1}^{1},\left(Z_{1}-\mathbb{Z}_{1}^{1}\right)=\mathbb{Z}_{1}^{2}$, we can rewrite it as

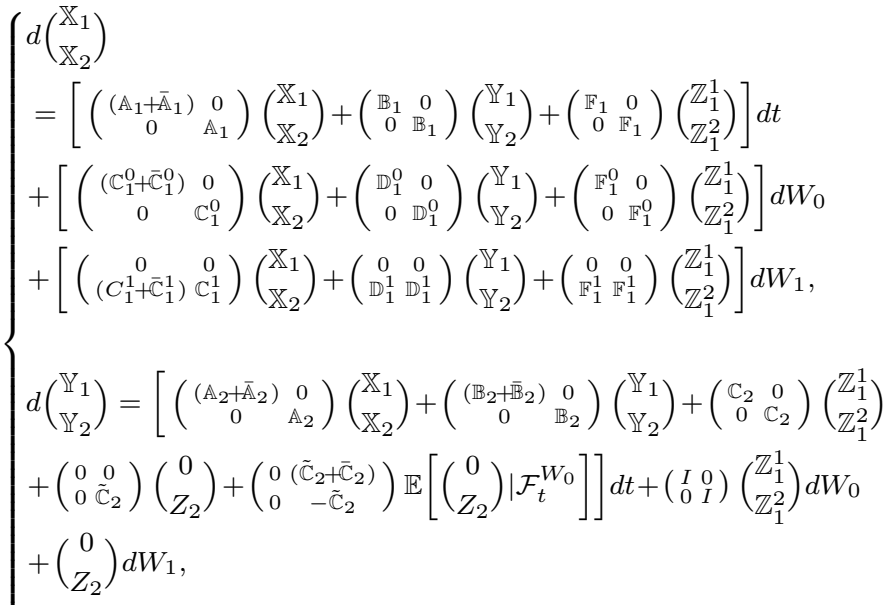

$$
\begin{aligned}
& X(0)=\left(\xi_{0}^{\top}, \xi^{\top}\right)^{\top}, \quad Y(T)=\left(0^{\top}, 0^{\top}, 0^{\top}, 0^{\top}, 0^{\top}\right)^{\top} .
\end{aligned}
$$

By letting

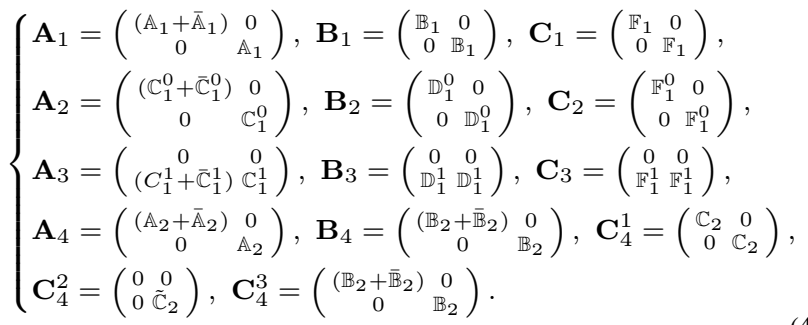

We have

$$
\begin{aligned}
& \int d\left(\begin{array}{l}
\mathbb{X}_{1} \\
\mathbb{X}_{2}
\end{array}\right)=\left[\mathbf{A}_{1}\left(\begin{array}{l}
\mathbb{X}_{1} \\
\mathbb{X}_{2}
\end{array}\right)+\mathbf{B}_{1}\left(\begin{array}{c}
\mathbb{Y}_{1} \\
\mathbb{Y}_{2}
\end{array}\right)+\mathbf{C}_{1}\left(\begin{array}{l}
\mathbb{Z}_{1}^{1} \\
\mathbb{Z}_{1}^{2}
\end{array}\right)\right] d t \\
& +\left[\mathbf{A}_{2}\left(\begin{array}{l}
\mathbb{X}_{1} \\
\mathbb{X}_{2}
\end{array}\right)+\mathbf{B}_{2}\left(\begin{array}{c}
\mathbb{Y}_{1} \\
\mathbb{Y}_{2}
\end{array}\right)+\mathbf{C}_{2}\left(\begin{array}{l}
\mathbb{Z}_{1}^{1} \\
\mathbb{Z}_{1}^{2}
\end{array}\right)\right] d W_{0} \\
& +\left[\mathbf{A}_{3}\left(\begin{array}{l}
\mathbb{X}_{1} \\
\mathbb{X}_{2}
\end{array}\right)+\mathbf{B}_{3}\left(\begin{array}{c}
\mathbb{Y}_{1} \\
\mathbb{Y}_{2}
\end{array}\right)+\mathbf{C}_{3}\left(\begin{array}{c}
\mathbb{Z}_{1}^{1} \\
\mathbb{Z}_{1}^{2}
\end{array}\right)\right] d W_{1} \\
& d\left(\begin{array}{c}
\mathbb{Y}_{1} \\
\mathbb{Y}_{2}
\end{array}\right)=\left[\mathbf{A}_{4}\left(\begin{array}{c}
\mathbb{X}_{1} \\
\mathbb{X}_{2}
\end{array}\right)+\mathbf{B}_{4}\left(\begin{array}{c}
\mathbb{Y}_{1} \\
\mathbb{Y}_{2}
\end{array}\right)+\mathbf{C}_{4}^{1}\left(\begin{array}{l}
\mathbb{Z}_{1}^{1} \\
\mathbb{Z}_{1}^{2}
\end{array}\right)+\mathbf{C}_{4}^{2}\left(\begin{array}{c}
0 \\
Z_{2}
\end{array}\right)\right. \\
& \left.+\mathbf{C}_{4}^{3} \mathbb{E}\left[\left(\begin{array}{c}
0 \\
Z_{2}
\end{array}\right) \mid \mathcal{F}_{t}^{W_{0}}\right]\right] d t+\left(\begin{array}{cc}
I & 0 \\
0 & I
\end{array}\right)\left(\begin{array}{l}
\mathbb{Z}_{1}^{1} \\
\mathbb{Z}_{1}^{2}
\end{array}\right) d W_{0}+\left(\begin{array}{c}
0 \\
Z_{2}
\end{array}\right) d W_{1} \\
& X(0)=\left(\xi_{0}^{\top}, \xi^{\top}\right)^{\top}, Y(T)=\left(0^{\top}, 0^{\top}, 0^{\top}, 0^{\top}, 0^{\top}\right)^{\top} .
\end{aligned}
$$

We can introduce the following Riccati equation

$$
\left\{\begin{array}{l}
\dot{\mathbf{K}}+\mathbf{K} \mathbf{A}_{1}+\mathbf{K B} \mathbf{B}_{1} \mathbf{K}-\mathbf{A}_{4}-\mathbf{B}_{4} \mathbf{K}+\left(\mathbf{K C}_{1}-\mathbf{C}_{4}\right) \\
\times\left(\mathbf{I}-\mathbf{K C}_{2}\right)^{-1}\left(\mathbf{K} \mathbf{A}_{2}+\mathbf{K} \mathbf{B}_{2} \mathbf{K}\right)=0 \\
\mathbf{K}(T)=0
\end{array}\right.
$$

Then it is easy to verify that

$$
\left\{\begin{array}{l}
\left(\begin{array}{c}
\mathbb{Y}_{1} \\
\mathbb{Y}_{2}
\end{array}\right)=\mathbf{K}\left(\begin{array}{l}
\mathbb{X}_{1} \\
\mathbb{X}_{2}
\end{array}\right), \\
\left(\begin{array}{l}
\mathbb{Z}_{1}^{1} \\
\mathbb{Z}_{1}^{2}
\end{array}\right)=\left(\mathbf{I}-\mathbf{K C}_{2}\right)^{-1}\left(\mathbf{K A}_{2}+\mathbf{K B}_{2} \mathbf{K}\right)\left(\begin{array}{l}
\mathbb{X}_{1} \\
\mathbb{X}_{2}
\end{array}\right), \\
\left(\begin{array}{c}
0 \\
Z_{2}
\end{array}\right)=\left(\mathbf{K A}_{3}+\mathbf{K B}_{3} \mathbf{K}\right)\left(\begin{array}{l}
\mathbb{X}_{1} \\
\mathbb{X}_{2}
\end{array}\right)+\mathbf{K C}_{3}\left(\begin{array}{l}
\mathbb{Z}_{1}^{1} \\
\mathbb{Z}_{1}^{2}
\end{array}\right) .
\end{array}\right.
$$

Then (44) becomes a decoupled BSDE

$$
\left\{\begin{array}{l}
d\left(\begin{array}{l}
\mathbb{X}_{1} \\
\mathbb{X}_{2}
\end{array}\right)=\left[\mathbf{A}_{1}+\mathbf{B}_{1} \mathbf{K}+\mathbf{C}_{1}\left(\mathbf{I}-\mathbf{K C}_{2}\right)^{-1}\left(\mathbf{K} \mathbf{A}_{2}+\mathbf{K} \mathbf{B}_{2} \mathbf{K}\right)\right]\left(\begin{array}{l}
\mathbb{X}_{1} \\
\mathbb{X}_{2}
\end{array}\right) d t \\
+\left[\mathbf{A}_{2}+\mathbf{B}_{2} \mathbf{K}+\mathbf{C}_{2}\left(\mathbf{I}-\mathbf{K C}_{2}\right)^{-1}\left(\mathbf{K} \mathbf{A}_{2}+\mathbf{K} \mathbf{B}_{2} \mathbf{K}\right)\right]\left(\begin{array}{l}
\mathbb{X}_{1} \\
\mathbb{X}_{2}
\end{array}\right) d W_{0} \\
+\left[\mathbf{A}_{3}+\mathbf{B}_{3} \mathbf{K}+\mathbf{C}_{3}\left(\mathbf{I}-\mathbf{K} \mathbf{C}_{2}\right)^{-1}\left(\mathbf{K} \mathbf{A}_{2}+\mathbf{K} \mathbf{B}_{2} \mathbf{K}\right)\right]\left(\begin{array}{l}
\mathbb{X}_{1} \\
\mathbb{X}_{2}
\end{array}\right) d W_{1}, \\
X(0)=\left(\xi_{0}^{\top}, \xi^{\top}\right)^{\top} .
\end{array}\right.
$$

Then by using Euler-Maruyama method, Milstein method and Runge-Kutta method, $\left(\hat{x}, z_{0}, y_{1}^{0}, \beta_{1}^{0}, \hat{y}_{1}, \hat{\beta}_{1}, y_{2}\right)$ can be obtained. Further, by (10) and (26), we can calculate $\Theta_{1}$, $\Theta_{2}, \Lambda_{1}, \Lambda_{2}$. Then, the realized states can be obtained by (1) and (2). The following graphs are the first coordinate of the 
realized states.

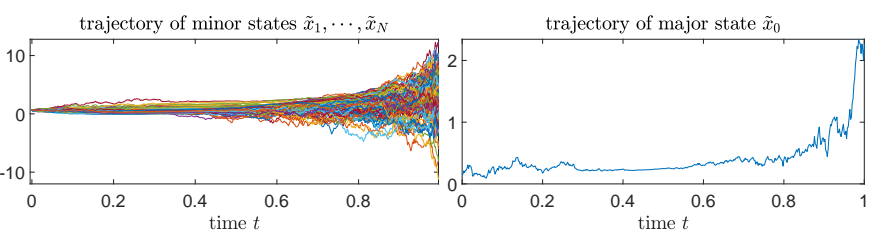

Then, the corresponding feedback form mean-field controls can be obtained as well. The following graphs are the first coordinate of the mean-field controls.

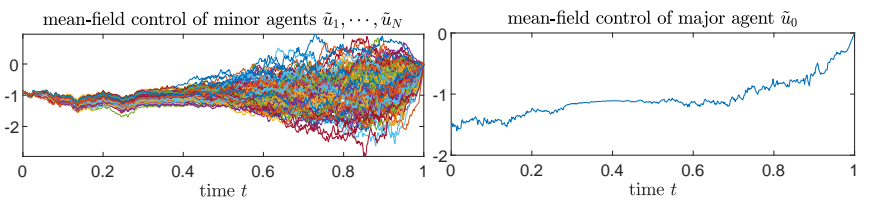

Next, we simulate the convergence of the population state average $\check{x}^{(N)}(t)$ to the mean field $\hat{x}$. Specifically, we will calculate $\mathbb{E} \sup _{0<t<T}\left\|\check{x}^{(N)}(t)-\hat{x}\right\|^{2}$. First, $\sup _{0<t<T}\left\|\check{x}^{(N)}(t)-\hat{x}\right\|^{2}$ can be calculated directly. Second, for the expectation, we repeat such process enough times (200 times) and take the average to simulate it.

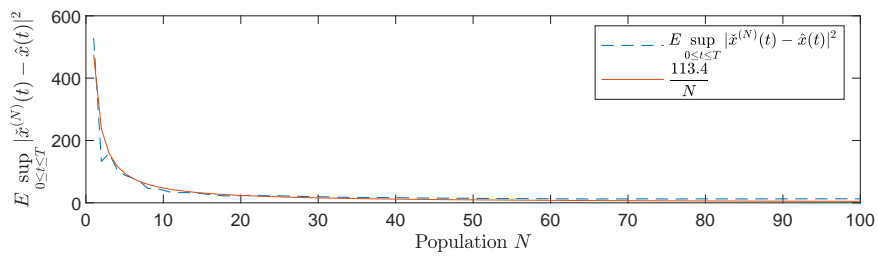

The relation between $\mathbb{E} \sup _{0 \leq t \leq T}\left\|\check{x}^{(N)}(t)-\hat{x}\right\|^{2}$ and $N$ can be fitted by $\mathbb{E} \sup _{0 \leq t \leq T}\left\|\check{x}^{(N)}(t)-\hat{x}\right\|^{2}=\frac{113.4}{N}$ with R-square 0.9944 . In this sense, $\mathbb{E} \sup _{0 \leq t \leq T}\left\|\check{x}^{(N)}(t)-\hat{x}\right\|^{2}=O\left(\frac{1}{N}\right)$.

By the simulation above, we can see that the mean-field strategy is asymptotically optimal.

\section{REFERENCES}

[1] F . Bloch, "Sequential Formation of Coalitions in Games with Externalities and Fixed Payoff Division[J]," Games and Economic Behavior, vol. 14, no. 1, pp. 90-123, 1996.

[2] A. Bensoussan, K. C. J. Sung, S. C. P. Yam, and S.-P. Yung, "Linearquadratic mean field games," J. Optim. Theory Appl., vol. 169, no. 2, pp. 496-529, 2016.

[3] R. Carmona and F. Delarue, "Probabilistic Analysis of Mean-Field Games," SIAM J. Control Optim., vol. 51, no. 4, pp. 2705-2734, Jan. 2013.

[4] R. Carmona and P. Wang, "An alternative approach to mean field game with major and minor players, and applications to herders impacts," Applied Mathematics \& Optimization, vol. 76, no. 1, pp. 5-27, 2017.

[5] R. Carmona and X. Zhu, "A probabilistic approach to mean field games with major and minor players," Ann. Appl. Probab., vol. 26, no. 3, pp. 1535-1580, Jun. 2016, Accessed: Aug. 21, 2020.

[6] H. Fang, Y. Wang, and J. Chen, "Health-aware and user-involved battery charging management for electric vehicles: Linear quadratic strategies," IEEE Transactions on Control Systems Technology, vol. 25, no. 3, pp. 911-923, 2016.

[7] L. Gan, U. Topcu, and S. Low. "Optimal Decentralized Protocol for Electric Vehicle Charging," IEEE Trans. Power Sys., vol. 28, no, 2, pp. 940-951, 2013.

[8] P.J. Graber, "Linear Quadratic Mean Field Type Control and Mean Field Games with Common Noise, with Application to Production of an Exhaustible Resource," Appl. Math Optim., vol. 74, pp. 459-486, 2016.

[9] S. Hart, and M. Kurz, "Endogenous formation of coalitions," Econometrica: Journal of the econometric society, pp. 1047-1064, 1983.

[10] Y. Hu, J. Huang, and X. Li, "Linear quadratic mean field game with control input constraint," ESAIM Control Optim. Calc. Var., vol. 24, no. 2, pp. 901-919, 2018, Accessed: Aug. 10, 2020.
[11] Y. Hu, J. Huang, and T. Nie, "Linear-Quadratic-Gaussian Mixed MeanField Games with Heterogeneous Input Constraints," SIAM J. Control Optim., vol. 56, no. 4, pp. 2835-2877, Jan. 2018.

[12] J. Huang, B. Wang, and J. Yong, "Social Optima in Mean Field LinearQuadratic-Gaussian Control with Volatility Uncertainty," arXiv preprint arXiv:1912.06371 2019.

[13] J. Huang, S. Wang, and Z. Wu, "Backward Mean-Field LinearQuadratic-Gaussian (LQG) Games: Full and Partial Information," IEEE Trans. Automat. Contr., vol. 61, no. 12, pp. 3784-3796, Dec. 2016.

[14] M. Huang, "Large-Population LQG Games Involving a Major Player: The Nash Certainty Equivalence Principle," SIAM J. Control Optim., vol. 48, no. 5, pp. 3318-3353, Jan. 2010.

[15] M. Huang, P. E. Caines, and R. P. Malhame, "Large-Population CostCoupled LQG Problems With Nonuniform Agents: Individual-Mass Behavior and Decentralized $\varepsilon$-Nash Equilibria,” IEEE Trans. Automat. Contr., vol. 52, no. 9, pp. 1560-1571, Sep. 2007.

[16] M. Huang, P. E. Caines, and R. P. Malhame, "Social Optima in Mean Field LQG Control: Centralized and Decentralized Strategies," IEEE Trans. Automat. Contr., vol. 57, no. 7, pp. 1736-1751, Jul. 2012.

[17] M. Huang, P. E. Caines, and R. P. Malhame, "Mean Field Games," Handbook of Dynamic Game Theory. Springer, 2015.

[18] J.-M. Lasry and P.-L. Lions, "Mean field games," Jpn. J. Math., vol. 2, no. 1, pp. 229-260, 2007

[19] Z. Ma, D. Callaway, and I. Hiskens, "Decentralized charging control of large populations of plug-in electric vehicles," IEEE Trans. Con. Sys. Tech., vol. 21, no. 1, pp. 67-78, 2013.

[20] J. Moon and T. Basar. Discrete-time LQG mean field games with unreliable communication. 53rd IEEE Conference on Decision and Control, (2015), 2697-2702.

[21] J. Moon and T. Basar. Risk-sensitive mean field games via the stochastic maximum principle. Dynamic Games and Applications, (2018), 1-26.

[22] Y. Hu, J. Huang, and T. Nie. "Linear-quadratic-gaussian mixed meanfield games with heterogeneous input constraints." SIAM J. Control Optim., vol. 56, no. 4, pp. 2835-2877, 2018.

[23] M. Nourian and P. E. Caines, " $\varepsilon$-Nash Mean Field Game Theory for Nonlinear Stochastic Dynamical Systems with Major and Minor Agents," SIAM J. Control Optim., vol. 51, no. 4, pp. 3302-3331, Jan. 2013.

[24] M. Nourian, P. E. Caines, R. P. Malhame and M. Huang, "Nash, Social and Centralized Solutions to Consensus Problems via Mean Field Control Theory," in IEEE Transactions on Automatic Control, vol. 58 no. 3, pp. 639-653, March 2013, doi: 10.1109/TAC.2012.2215399.

[25] Z. Qiu, J. Huang, and T. Xie, "Linear Quadratic Gaussian Mean-Field Controls of Social Optima," arXiv preprint arXiv:2005.06792 2019.

[26] D. Ray and R. Vohra, "A theory of endogenous coalition structures," Games and economic behavior, vol. 26, no. 2, pp. 286-336, 1999.

[27] J. Sun, X. Li, and J. Yong, "Open-Loop and Closed-Loop Solvabilities for Stochastic Linear Quadratic Optimal Control Problems," SIAM Journal on Control and Optimization, vol. 54, no. 5. pp. 2274-2308, 2016.

[28] B.-C. Wang and J. Huang, "Social optima in robust mean field LQG control," 2017 11th Asian Control Conference (ASCC), 2017.

[29] J. Yong and X. Y. Zhou, Stochastic Controls: Hamiltonian Systems and HJB Equations. Springer Science \& Business Media, 1999.

\section{AppendiX}

For any given $(Y, Z) \in L_{\mathcal{F}}^{2}\left(0, T ; \mathbb{R}^{m}\right) \times L_{\mathcal{F}}^{2}\left(0, T ; \mathbb{R}^{m \times(d+1)}\right)$ and $0 \leq t \leq T$, the following $\mathrm{SDE}$ has a unique solution:

$$
\begin{aligned}
X(t)= & +\int_{0}^{t} b\left(s, X(s), \mathbb{E}^{W_{0}}[X(s)], Y(s), Z(s)\right) d s \\
& +\int_{0}^{t} \sigma\left(s, X(s), \mathbb{E}^{W_{0}}[X(s)], Y(s), Z(s)\right) d W(s) .
\end{aligned}
$$

Therefore, we can introduce a map $\mathcal{M}_{1}:(Y, Z) \in$ $L_{\mathcal{F}}^{2}\left(0, T ; \mathbb{R}^{m}\right) \times L_{\mathcal{F}}^{2}\left(0, T ; \mathbb{R}^{m \times(d+1)}\right) \rightarrow X \in L_{\mathcal{F}}^{2}\left(0, T ; \mathbb{R}^{n}\right)$ by (46). Moreover, we have the following result:

Lemma 10: Let $X_{i}$ be the solution of (46) corresponding to $\left(Y_{i}, Z_{i}\right), i=1,2$ respectively. Then for all $\rho \in \mathbb{R}$ and some 
constant $l_{1}>0$, we have

$$
\begin{aligned}
& \mathbb{E} e^{-\rho t}\|\hat{X}(t)\|^{2}+\bar{\rho}_{1} \mathbb{E} \int_{0}^{t} e^{-\rho s}\|\hat{X}(s)\|^{2} d s \\
\leq & \left(k_{2} l_{1}+k_{11}^{2}\right) \mathbb{E} \int_{0}^{t} e^{-\rho s}\|\hat{Y}(s)\|^{2} d s+\left(k_{3} l_{2}+k_{12}^{2}\right) \mathbb{E} \int_{0}^{t} e^{-\rho s}\|\hat{Z}(s)\|^{2} d s,
\end{aligned}
$$

and

$$
\begin{aligned}
\mathbb{E} e^{-\rho t}\|\hat{X}(t)\|^{2} \leq & \left(k_{2} l_{1}+k_{11}^{2}\right) \mathbb{E} \int_{0}^{t} e^{-\bar{\rho}_{1}(t-s)-\rho s}\|\hat{Y}(s)\|^{2} d s \\
& +\left(k_{2} l_{2}+k_{12}^{2}\right) \mathbb{E} \int_{0}^{t} e^{-\bar{\rho}_{1}(t-s)-\rho s}\|\hat{Z}(s)\|^{2} d s
\end{aligned}
$$

where $\bar{\rho}_{1}=\rho-2 \rho_{1}-2 k_{1}-k_{2} l_{1}^{-1}-k_{3} l_{2}^{-1}-k_{9}^{2}-k_{10}^{2}$ and $\hat{\Phi}=\Phi_{1}-\Phi_{2}, \Phi=X, Y, Z$. Moreover,

$$
\begin{aligned}
& \mathbb{E} \int_{0}^{T} e^{-\rho t}\|\hat{X}(t)\|^{2} d t \\
\leq & \frac{1-e^{-\bar{\rho}_{1} T}}{\bar{\rho}_{1}}\left[\left(k_{2} l_{1}+k_{11}^{2}\right) \mathbb{E} \int_{0}^{t} e^{-\rho t}\|\hat{Y}(t)\|^{2} d t\right. \\
& \left.+\left(k_{3} l_{2}+k_{12}^{2}\right) \mathbb{E} \int_{0}^{t} e^{-\rho t}\|\hat{Z}(t)\|^{2} d t\right],
\end{aligned}
$$

and

$$
\begin{aligned}
e^{-\rho T} \mathbb{E}\|\hat{X}(T)\|^{2} \leq & \left(1 \vee e^{-\bar{\rho}_{1} T}\right)\left[\left(k_{2} l_{1}+k_{11}^{2}\right) \mathbb{E} \int_{0}^{T} e^{-\rho t}\|\hat{Y}(t)\|^{2} d t\right. \\
& \left.+\left(k_{3} l_{2}+k_{12}^{2}\right) \mathbb{E} \int_{0}^{T} e^{-\rho t}\|\hat{Z}(t)\|^{2} d t\right] .
\end{aligned}
$$

Specifically, if $\bar{\rho}_{1}>0$,

$$
\begin{aligned}
e^{-\rho T} \mathbb{E}\|\hat{X}(T)\|^{2} \leq & \left(k_{2} l_{1}+k_{11}^{2}\right) \mathbb{E} \int_{0}^{T} e^{-\rho t}\|\hat{Y}(t)\|^{2} d t \\
& +\left(k_{3} l_{2}+k_{12}^{2}\right) \mathbb{E} \int_{0}^{T} e^{-\rho t}\|\hat{Z}(t)\|^{2} d t .
\end{aligned}
$$

Proof: For any $\rho>0$, applying Itô's formula to $e^{-\rho t}\|\hat{X}(t)\|^{2}$

$$
\begin{aligned}
& \mathbb{E} e^{-\rho t}\|\hat{X}(t)\|^{2}+\rho \mathbb{E} \int_{0}^{t} e^{-\rho s}\|\hat{X}(s)\|^{2} d s \\
&= 2 \mathbb{E} \int_{0}^{t} e^{-\rho s} \hat{X}(s)\left(b\left(s, X_{1}(s), \mathbb{E}^{W_{0}}\left[X_{1}(s)\right], Y_{1}(s), Z_{1}(s)\right)\right. \\
&\left.-b\left(s, X_{2}(s), \mathbb{E}^{W_{0}}\left[X_{2}(s)\right], Y_{2}(s), Z_{2}(s)\right)\right) d s \\
&+\mathbb{E} \int_{0}^{t} e^{-\rho s}\left(\sigma\left(s, X_{1}(s), \mathbb{E}^{W_{0}}\left[X_{1}(s)\right], Y_{1}(s), Z_{1}(s)\right)\right.\left.\quad-\sigma\left(s, X_{2}(s), \mathbb{E}^{W_{0}}\left[X_{2}(s)\right], Y_{2}(s), Z_{2}(s)\right)\right)^{2} d s \\
& \leq \mathbb{E} \int_{0}^{t} e^{-\rho s}\left[\left(2 \rho_{1}+2 k_{1}+k_{2} l_{1}^{-1}+k_{3} l_{2}^{-1}+k_{9}^{2}+k_{10}^{2}\right)\|\hat{X}(s)\|^{2}\right. \\
&\left.\quad+\left(k_{2} l_{1}+k_{11}^{2}\right)\|\hat{Y}(s)\|^{2}+\left(k_{3} l_{2}+k_{12}^{2}\right)\|\hat{Z}(s)\|^{2}\right] d s .
\end{aligned}
$$

Similarly, applying Itô's formula to $e^{-\bar{\rho}_{1}(t-s)-\rho s}\|\hat{X}(s)\|^{2}$, we have (48). Integrating from 0 to $T$ on both sides of (48) and noting that $\frac{1-e^{-\bar{\rho}_{1}(t-s)}}{\bar{\rho}_{1}} \leq \frac{1-e^{-\bar{\rho}_{1} T}}{\bar{\rho}_{1}}$, we have

$$
\begin{aligned}
& \mathbb{E} \int_{0}^{T} e^{-\rho t}\|\hat{X}(t)\|^{2} d t \\
\leq & \left(k_{2} l_{1}+k_{11}^{2}\right) \frac{1-e^{-\bar{\rho}_{1} T}}{\bar{\rho}_{1}} \mathbb{E} \int_{0}^{T} e^{-\rho s}\|\hat{Y}(s)\|^{2} d s \\
& +\left(k_{3} l_{2}+k_{12}^{2}\right) \frac{1-e^{-\bar{\rho}_{1} T}}{\bar{\rho}_{1}} \mathbb{E} \int_{0}^{T} e^{-\rho s}\|\hat{Z}(s)\|^{2} d s .
\end{aligned}
$$

Letting $t=T$ in (48), we have (50).

For any given $X \in L_{\mathcal{F}}^{2}\left(0, T ; \mathbb{R}^{n}\right)$, the following BSDE has a unique solution:

$$
\begin{array}{r}
Y(t)=\int_{t}^{T} f\left(s, X(s), \mathbb{E}^{W_{0}}[X(s)], Y(s), \mathbb{E}^{W_{0}}[Y(s)],\right. \\
\left.Z(s), \mathbb{E}^{W_{0}}[Z(s)]\right) d s-\int_{t}^{T} Z(s) d W(s) .
\end{array}
$$

Thus, we can introduce another map $\mathcal{M}_{2}: X \in L_{\mathcal{F}}^{2}\left(0, T ; \mathbb{R}^{n}\right) \rightarrow$ $(Y, Z) \in L_{\mathcal{F}}^{2}\left(0, T ; \mathbb{R}^{m}\right) \times L_{\mathcal{F}}^{2}\left(0, T ; \mathbb{R}^{m \times(d+1)}\right)$ by (52). Similarly, we have the following result:

Lemma 11: Let $\left(Y_{i}, Z_{i}\right)$ be the solution of (52) corresponding to $X_{i}, i=1,2$, respectively. Then for all $\rho \in \mathbb{R}$ and some constants $l_{3}, l_{4}, l_{5}, l_{6}>0$ such that

$$
\begin{aligned}
& \mathbb{E} e^{-\rho t}\|\hat{Y}(t)\|^{2}+\bar{\rho}_{2} \mathbb{E} \int_{t}^{T} e^{-\rho s}\|\hat{Y}(s)\|^{2} d s \\
& +\left(1-k_{7} l_{5}-k_{8} l_{6}\right) \mathbb{E} \int_{t}^{T} e^{-\rho s}\|\hat{Z}(s)\|^{2} d s \\
& \leq\left(k_{4} l_{3}+k_{5} l_{4}\right) \mathbb{E} \int_{t}^{T} e^{-\rho s}\|\hat{X}(s)\|^{2} d s,
\end{aligned}
$$

and

$$
\begin{aligned}
& \mathbb{E} e^{-\rho t}\|\hat{Y}(t)\|^{2}+\left(1-k_{7} l_{5}-k_{8} l_{6}\right) \mathbb{E} \int_{t}^{T} e^{-\rho s}\|\hat{Z}(s)\|^{2} d s \\
\leq & \left(k_{4} l_{3}+k_{5} l_{4}\right) \mathbb{E} \int_{t}^{T} e^{-\bar{\rho}_{2}(s-t)-\rho s}\|\hat{X}(s)\|^{2} d s,
\end{aligned}
$$

where $\bar{\rho}_{2}=-\rho-2 \rho_{2}-2 k_{6}-k_{4} l_{3}^{-1}-k_{5} l_{4}^{-1}-k_{7} l_{5}^{-1}-k_{8} l_{6}^{-1}$, and $\hat{\Phi}=\Phi_{1}-\Phi_{2}, \Phi=X, Y, Z$. Moreover,

$\mathbb{E} \int_{0}^{T} e^{-\rho t}\|\hat{Y}(t)\|^{2} d t \leq \frac{1-e^{-\bar{\rho}_{2} T}}{\bar{\rho}_{2}}\left(k_{4} l_{3}+k_{5} l_{4}\right) \mathbb{E} \int_{0}^{T} e^{-\rho s}\|\hat{X}(s)\|^{2} d s$,

and

$$
\begin{aligned}
& \mathbb{E} \int_{0}^{T} e^{-\rho t}\|\hat{Z}(t)\|^{2} d t \\
\leq & \frac{\left(k_{4} l_{3}+k_{5} l_{4}\right)\left(1 \vee e^{-\bar{\rho}_{2} T}\right)}{\left(1-k_{7} l_{5}-k_{8} l_{6}\right)\left(1 \wedge e^{-\bar{\rho}_{2} T}\right)} \mathbb{E} \int_{0}^{T} e^{-\rho s}\|\hat{X}(s)\|^{2} d s .
\end{aligned}
$$

Specifically, if $\bar{\rho}_{2}>0$,

$\mathbb{E} \int_{0}^{T} e^{-\rho t}\|\hat{Z}(t)\|^{2} d t \leq \frac{k_{4} l_{3}+k_{5} l_{4}}{1-k_{7} l_{5}-k_{8} l_{6}} \mathbb{E} \int_{0}^{T} e^{-\rho s}\|\hat{X}(s)\|^{2} d s$. Proof of Theorem 1; Define $\mathcal{M}:=\mathcal{M}_{2} \circ \mathcal{M}_{1}$, where $\mathcal{M}_{1}$ is defined by (46) and $\mathcal{M}_{2}$ is defined by (52). Thus, $\mathcal{M}$ is a mapping from $L_{\mathcal{F}}^{2}\left(0, T ; \mathbb{R}^{m}\right) \times L_{\mathcal{F}}^{2}\left(0, T ; \mathbb{R}^{m \times(d+1)}\right)$ into itself. For $\left(U_{i}, V_{i}\right) \in L_{\mathcal{F}}^{2}\left(0, T ; \mathbb{R}^{m}\right) \times L_{\mathcal{F}}^{2}\left(0, T ; \mathbb{R}^{m \times(d+1)}\right)$, let $X_{i}:=$ $\mathcal{M}_{1}\left(U_{i}, V_{i}\right)$ and $\left(Y_{i}, Z_{i}\right):=\mathcal{M}\left(U_{i}, V_{i}\right)$. Therefore, 


$$
\begin{aligned}
& \mathbb{E} \int_{0}^{T} e^{-\rho t}\left\|Y_{1}(t)-Y_{2}(t)\right\|^{2} d t+\mathbb{E} \int_{0}^{T} e^{-\rho t}\left\|Z_{1}(t)-Z_{2}(t)\right\|^{2} d t \\
\leq & {\left[\frac{1-e^{-\bar{\rho}_{2} T}}{\bar{\rho}_{2}}+\frac{1 \vee e^{-\bar{\rho}_{2} T}}{\left(1-k_{7} l_{5}-k_{8} l_{6}\right)\left(1 \wedge e^{-\bar{\rho}_{2} T}\right)}\right]\left(k_{4} l_{3}+k_{5} l_{4}\right) } \\
& \times \mathbb{E} \int_{0}^{T} e^{-\rho t}\left\|X_{1}(t)-X_{2}(t)\right\|^{2} d t \\
\leq & {\left[\frac{1-e^{-\bar{\rho}_{2} T}}{\bar{\rho}_{2}}+\frac{1 \vee e^{-\bar{\rho}_{2} T}}{\left(1-k_{7} l_{5}-k_{8} l_{6}\right)\left(1 \wedge e^{-\bar{\rho}_{2} T}\right)}\right] \frac{1-e^{-\bar{\rho}_{1} T}}{\bar{\rho}_{1}} } \\
& \times\left(k_{4} l_{3}+k_{5} l_{4}\right)\left[\left(k_{2} l_{1}+k_{11}^{2}\right) \mathbb{E} \int_{0}^{T} e^{-\rho t}\left\|U_{1}(t)-U_{2}(t)\right\|^{2} d t\right. \\
& \left.+\left(k_{3} l_{2}+k_{12}^{2}\right) \mathbb{E} \int_{0}^{T} e^{-\rho t}\left\|V_{1}(t)-V_{2}(t)\right\|^{2} d t\right] .
\end{aligned}
$$

Choosing suitable $\rho$, we get that $\mathcal{M}$ is a contraction mapping.

Furthermore, if $2 \rho_{1}+2 \rho_{2}<-2 k_{1}-2 k_{6}-2 k_{7}^{2}-2 k_{8}^{2}-k_{9}^{2}-$ $k_{10}^{2}$, we can choose $\rho \in \mathbb{R}, 0<k_{7} l_{5}<\frac{1}{2}$ and $0<k_{8} l_{6}<\frac{1}{2}$ and sufficient large $l_{1}, l_{2}, l_{3}, l_{4}$ such that

$$
\bar{\rho}_{1}>0, \quad \bar{\rho}_{2}>0, \quad 1-k_{7} l_{5}-k_{8} l_{6}>0 .
$$

Therefore,

$$
\begin{aligned}
& \quad \mathbb{E} \int_{0}^{T} e^{-\rho t}\left\|Y_{1}(t)-Y_{2}(t)\right\|^{2} d t+\mathbb{E} \int_{0}^{T} e^{-\rho t}\left\|Z_{1}(t)-Z_{2}(t)\right\|^{2} d t \\
& \leq\left[\frac{1}{\bar{\rho}_{2}}+\frac{1}{1-k_{7} l_{5}-k_{8} l_{6}}\right] \frac{1}{\bar{\rho}_{1}}\left(k_{4} l_{3}+k_{5} l_{4}\right) \\
& \quad \times\left[\left(k_{2} l_{1}+k_{11}^{2}\right) \mathbb{E} \int_{0}^{T} e^{-\rho t}\left\|U_{1}(t)-U_{2}(t)\right\|^{2} d t\right. \\
& \left.\quad+\left(k_{3} l_{2}+k_{12}^{2}\right) \mathbb{E} \int_{0}^{T} e^{-\rho t}\left\|V_{1}(t)-V_{2}(t)\right\|^{2} d t\right] .
\end{aligned}
$$

The proof is complete. 